\title{
Weighted Matching Markets with Budget Constraints
}

\author{
Anisse Ismaili \\ RIKEN Center for Advanced Intelligence Project \\ Naoto Hamada \\ Graduate School of Information Science and \\ Electrical Engineering, Kyushu University \\ Yuzhe Zhang \\ Bernoulli Institute, University of Groningen \\ Takamasa Suzuki \\ Gifu Shotoku Gakuen University \\ Makoto Yokoo \\ Graduate School of Information Science and \\ Electrical Engineering, Kyushu University
}

ANISSE.ISMAILI@RIKEN.JP

HAMADA@AGENT.INF.KYUSHU-U.AC.JP

YOEZY.ZHANG@RUG.NL

T.SUZUKI@GIFU.SHOTOKU.AC.JP

YOKOO@INF.KYUSHU-U.AC.JP

\begin{abstract}
We investigate markets with a set of students on one side and a set of colleges on the other. A student and college can be linked by a weighted contract that defines the student's wage, while a college's budget for hiring students is limited. Stability is a crucial requirement for matching mechanisms to be applied in the real world. A standard stability requirement is coalitional stability, i.e., no pair of a college and group of students has any incentive to deviate. We find that a coalitionally stable matching is not guaranteed to exist, verifying the coalitional stability for a given matching is coNP-complete, and the problem of finding whether a coalitionally stable matching exists in a given market, is $\Sigma_{2}^{P}$-complete: $\mathrm{NP}^{\mathrm{NP}}$-complete. Other negative results also hold when blocking coalitions contain at most two students and one college. Given these computational hardness results, we pursue a weaker stability requirement called pairwise stability, where no pair of a college and single student has an incentive to deviate. Unfortunately, a pairwise stable matching is not guaranteed to exist either. Thus, we consider a restricted market called a typed weighted market, in which students are partitioned into types that induce their possible wages. We then design a strategy-proof and Pareto efficient mechanism that works in polynomial-time for computing a pairwise stable matching in typed weighted markets.
\end{abstract}

\section{Introduction}

Investigation into two-sided matchings began with Gale and Shapley (1962), who introduced the college admissions problem. Since then, the theory of two-sided matching and its applications to real-life problems have been extensively developed in the literature of economics (see Roth \& Sotomayor, 1990, for a comprehensive survey). This topic has recently attracted much attention from artificial intelligence and multi-agent systems as well (e.g. Goto et al., 2016; Kurata et al., 2017; Perrault et al., 2016). The problems of matching students to schools (Abdulkadiroğlu, Pathak, \& Roth, 2009; Abdulkadiroğlu, Pathak, Roth, \& Sönmez, 2005; Abdulkadiroğlu \& Sönmez, 2003), doctors to hospitals (Roth, 1984; Roth \& Peranson, 1999), and military cadets to army branches (Sönmez, 2013; Sönmez \& Switzer, 
2013) are important formal settings that have been considered. Central notions are pairwise and coalitional stability of a matching, which should be immune to deviations by a pair or group of agents. It is preferable for a mechanism to be student-wise strategy-proof: there should be no incentives for students ${ }^{1}$ to misreport their preferences.

The presence of maximum quotas (i.e. capacity limit of a college) is assumed in most standard models. In real-life examples, different kinds of distributional constraints exist other than maximum quotas (Biró, Fleiner, Irving, \& Manlove, 2010; Kamada \& Kojima, 2015), and various types of distributional constraints have recently been addressed and a series of mechanisms have been introduced to achieve desirable outcomes under such constraints (Fragiadakis, Iwasaki, Troyan, Ueda, \& Yokoo, 2015; Fragiadakis \& Troyan, 2017; Goto et al., 2016; Kojima, Tamura, \& Yokoo, 2018; Kurata et al., 2017). In this paper, we revisit the standard distributional constraint of maximum quotas by assuming that each college has a fixed amount of resource, or budget, that can be distributed among students and that students may receive a different amount. This amount may differ among different types of students (e.g., tuition at American state universities is lower for students who qualify for in-state rates), a student may be allocated a different amount of resource, depending on the contract she made (e.g., full or partial scholarship) or both. In our model, we explicitly take into account the total amount of each school's resources and the maximum a student may receive. Therefore, we model a weighted matching market with budget constraints.

Although our model is a natural extension of standard maximum quotas, very little literature has addressed this issue, possibly due to its intractability; two conditions from the literature, substitutability and the law of aggregate demand, make analysis tractable (Hatfield \& Milgrom, 2005), but neither is satisfied in our model. The most relevant work (Abizada, 2016) studies college admissions with budget constraints, in which a student receives a pair of a college and a stipend, and develops a strategy-proof mechanism that satisfies a weaker notion of stability. The major differences between this model and ours are that we deal with general ordinal (instead of quasi-linear) preferences of students, which becomes possible since we focus on discrete sets of wages (instead of a continuum) and allow different types of students to exist in a market (instead of assuming all students are of the same type). Another relevant work (Mongell \& Roth, 1986) shows that the core can be empty in a job market with budget constraints. We cannot apply their result to our model since they assume the utility of each school/firm is quasi-linear, while in our model, each school is indifferent about the amount of money it spends as long as it is below the budget limit. The environment that groups students into types has also been studied in the literature of school choice problems (Abdulkadiroğlu, 2005; Abdulkadiroğlu \& Sönmez, 2003; Ehlers, Hafalir, Yenmez, \& Yildirim, 2014; Kojima, 2012; Yenmez, Yildirim, \& Hafalir, 2013). Recently, a similar model was considered (Kawase \& Iwasaki, 2017, 2018). The goals of their works (approximate matching) are different from ours. While our model is more general, they concentrate on additive utilities of colleges. First, they relax feasibility such that a college is allowed to exceed its budget limit to some extent (Kawase \& Iwasaki, 2017). Second, they relax pairwise stability such that a college does not form a blocking pair unless its utility increases more than a certain factor $\alpha$ (Kawase \& Iwasaki, 2018).

1. The rest of this paper is described in the context of a college-student matching problem. 


\begin{tabular}{|c|c|c|}
\hline $\begin{array}{c}\text { Coalitional stability } \\
\text { (any blocking coalition) }\end{array}$ & $\begin{array}{c}\text { Blocking coalitions } \\
\text { with two students }\end{array}$ & $\begin{array}{c}\text { Pairwise } \\
\text { stability }\end{array}$ \\
\hline \multicolumn{2}{|c|}{$\begin{array}{c}\text { May not exist, even } \\
\text { in typed markets (Th. 1) }\end{array}$} & $\begin{array}{c}\text { May not exist in } \\
\text { general markets (Th. 6) }\end{array}$ \\
\hline \begin{tabular}{c|c} 
Verification is coNP-complete, \\
even in typed markets (Th. 2).
\end{tabular} & $\begin{array}{c}\text { Existence is } \\
\text { NP-hard, } \\
\text { Existence is NP }\end{array}$-complete \\
in general markets (Th. 3). & $\begin{array}{c}\text { even in typed } \\
\text { markets (Th. 5). }\end{array}$ & $\begin{array}{c}\text { strategy-proof, pairwise stable } \\
\text { and constrained Pareto efficient. }\end{array}$ \\
\hline
\end{tabular}

Table 1: Summary of our contributions

We also address computational issues related to verifying/finding a stable matching. To the best of our knowledge, we are the first to address these issues in two-sided matching with budget constraints. We show that coalitional stability in matchings with budget constraints involves a larger complexity class than NP in the polynomial hierarchy (Meyer \& Stockmeyer, 1972). According to a compendium of problems (updated in 2008), few $\Sigma_{2}^{P}$ complete (that is $\mathrm{NP}^{\mathrm{NP}}$-complete) problems involve numbers (Schaefer \& Umans, 2002). The $\forall \exists$ SubsetSum problem that we introduce (as a mid-step in our reduction) seems new. This compendium does not reflect the more recent progress in algorithmic game theory. The complexity of coalitional stability has been studied in several related models, in which checking is often coNP-complete and deciding coalitional stability is also $\Sigma_{2}^{P}$-complete. For instance, this is the case in additively separable hedonic games (Sung \& Dimitrov, 2007; Woeginger, 2013), for envy freeness (and Pareto efficiency) (Aziz, Brandt, \& Seedig, 2013) and in resource allocation (Bouveret \& Lang, 2008). Furthermore, NP-completeness of deciding whether a stable outcome exists was shown in matching problems with couples (Ronn, 1990) and matching problems with minimum quotas (Biró et al., 2010; Huang, 2010).

The contributions of this paper are twofold. First, we investigate the computational issues regarding the coalitional stability of a matching and find the following negative results. (1) There may not exist a coalitionally stable outcome. (2) Checking whether a given matching is coalitionally stable is coNP-complete. (3) It is $\mathrm{NP}^{\mathrm{NP}}$-complete to decide whether there exists a coalitionally stable matching. Assuming that blocking coalitions contain at most two students, (4) checking becomes polynomial and the existence problem is still NPcomplete. Therefore, the notion of coalitional stability is computationally intractable in typed weighted markets. Second, following the above results, we focus on pairwise stability, a weaker notion that involves only a pair of one student and a college. For a student, finding a profitable deviation in a group involving other students would be difficult, while finding such a deviation that involves only herself and a college would be much easier. Thus, we suggest that eliminating such deviation makes sense in practice. Unfortunately, we show that a pairwise stable matching is not guaranteed to exist in general. ${ }^{2}$ Thus, we consider a restricted market called a typed weighted market, in which students are partitioned into types that induce their possible wages. This result is surprising, since even in a typed weighted market, substitutability is not guaranteed to hold even for a typed weighted market. (Substitutability is a sufficient condition for the existence of a stable matching (Hatfield \& Kojima, 2010).) However, we show that there always exists a pairwise

2. This result is stark contrast to the model presented in (Abizada, 2016), in which a pairwise stable matching is guaranteed to exist. 
stable matching by developing a strategy-proof mechanism that finds such a matching in polynomial-time in a typed weighted market.

\section{Models}

Here, we present our two-sided weighted matchings models, where the most general is weighted markets. Simple weighted markets and typed weighted markets are particular cases. Our model is based on the framework of matching with contracts (Hatfield \& Milgrom, 2005), which is widely used in two-sided matching (Sönmez, 2013; Sönmez \& Switzer, 2013; Hatfield \& Kojima, 2008, 2010; Hatfield \& Kominers, 2009, 2012; Hatfield, Kominers, Nichifor, Ostrovsky, \& Westkamp, 2013; Echenique, 2012; Kominers \& Sönmez, 2016).

Definition 1. A weighted market is a tuple $\pi=\left(S, C, W, X, b_{C}, \succ_{S}, \tilde{\succsim}_{C}\right)$, where:

- $S=\left\{s_{1}, \ldots, s_{n}\right\}$ is a set of students.

- $C=\left\{c_{1}, \ldots, c_{m}\right\}$ is a set of colleges.

- $W=\left\{w_{1}, \ldots, w_{p}\right\}$ are non-negative integer wages.

- $X \subseteq\{(s, c, w) \mid s \in S, c \in C, w \in W\}$ is a set of possible contracts where contract $x=(s, c, w)$ means that student $s$ is assigned to college $c$ with wage $w$.

- $b_{C}=\left(b_{c} \in \mathbb{N}_{+}\right)_{c \in C}$ is a profile of colleges' budgets.

- $\succ_{S}=\left(\succ_{s}\right)_{s \in S}$ is a profile of student preferences $\succ_{s}$ over college-wage tuples $C \times W$ and an additional tuple $\left(c_{\emptyset}, 0\right)$ which means that she stays home with no wage. ${ }^{3}$ We assume that $w>w^{\prime}$ implies $(c, w) \succ_{s}\left(c, w^{\prime}\right)$

- $\tilde{\succsim}_{C}=\left(\tilde{\succsim}_{c}\right)_{c \in C}$ is a profile of college weak preferences over sets $S^{\prime} \subseteq 2^{S}$ of the students. Each college weak preference $\tilde{\succsim}_{c}$ is based on a weak preference $\succsim_{c}$ over the students and null student $s_{\emptyset}$. Weak preference $\succsim$ partitions into asymmetric part $\succ$ and symmetric part $\sim$. Here, $s \succ_{c} s^{\prime}$ means college $c$ strictly prefers $s$ over $s^{\prime}$ and $s \sim_{c} s^{\prime}$ means $c$ is indifferent between students $s$ and $s^{\prime}$. College preferences satisfy responsiveness; for every pair of students $s, s^{\prime} \in S$ and subset of students $S^{\prime} \subseteq S \backslash\left\{s, s^{\prime}\right\}$, it holds that:

$$
s \succsim_{c} s^{\prime} \Leftrightarrow S^{\prime} \cup\{s\} \tilde{\succsim}_{c} S^{\prime} \cup\left\{s^{\prime}\right\}
$$

which also implies $s \succ_{c} s^{\prime} \Leftrightarrow S^{\prime} \cup\{s\} \tilde{\succ}_{c} S^{\prime} \cup\left\{s^{\prime}\right\}$, since $\succ_{c}$ and $\check{\succ}_{c}$ are asymmetric parts. ${ }^{4}$ Also, for every subset of students $S^{\prime} \subsetneq S$ and student $s \in S \backslash S^{\prime}$,

$$
s \succsim_{c} s_{\emptyset} \Leftrightarrow S^{\prime} \cup\{s\} \tilde{\succsim}_{c} S^{\prime}
$$

holds, which similarly implies $s \succ_{c} s_{\emptyset} \Leftrightarrow S^{\prime} \cup\{s\} \tilde{\succ}_{c} S^{\prime}$.

A market is simple weighted if, between every student-college tuple, there exists at most one possible wage. In this simpler case, the set of contracts can be represented by

3. This definition allows for preference $(c, 2) \succ_{s}\left(c_{\emptyset}, 0\right) \succ_{s}(c, 1)$; the wage matters for the feasibility of the same college.

4. The same holds with symmetric parts $\sim_{c}$ and $\tilde{\sim}_{c}$. 
a bipartite graph between students on one side and colleges on the other side, and each possible student-college edge is weighted by the corresponding wage. Therefore, in simple weighted markets, notation $w$ can be abused in a functional manner $w: S \times C \rightarrow W$ where $w(s, c) \in W$ is the wage received by student $s$ for attending college $c$ and function $w$ is only defined on tuples $(s, c)$ for which there is a contract.

Furthermore, in the general setting, the market can be represented by a bipartite multigraph between students and colleges and possibly multiple edges between each studentcollege pair, corresponding to their possible contracts. The functional abuse for wages is then $w: S \times C \rightarrow 2^{W}$.

Weighted markets also admit typed weighted markets as a particular case in which students are partitioned into types.

- $\Theta=\left\{\theta_{1}, \ldots, \theta_{k}\right\}$ is a finite set of student types.

- Function $\tau: S \rightarrow \Theta$ maps each student to its type. We assume for students $s, s^{\prime} \in S$ such that $\theta_{i}=\tau(s), \theta_{j}=\tau\left(s^{\prime}\right), i<j$, if $s \succ_{c} s_{\emptyset}$ and $s^{\prime} \succ_{c} s_{\emptyset}$ hold, then $s \succ_{c} s^{\prime}$ holds. In other words, as long as college $c$ thinks both students $s$ and $s^{\prime}$ are strictly better than $s_{\emptyset}, c$ always prefers a student with the higher type. ${ }^{5}$

- Set $W$ is represented as $W=\bigcup_{c \in C} \bigcup_{\theta \in \Theta} W_{c, \theta}$, where $W_{c, \theta}$ is the set of wages that college $c$ can give to type $\theta$ students. Formally, for all $s \in S$, for all $c \in C$ such that $s \succ_{c} s_{\emptyset}$, and for all $w \in W,(s, c, w) \in X$ holds if and only if $w \in W_{c, \tau(s)}$ holds. We assume types are ordered in the following sense. Given a college $c$, for every $w \in W_{c, \theta_{i}}$ and $w^{\prime} \in W_{c, \theta_{i+1}}$, one has $w>w^{\prime}$.

Definition 2. A typed weighted market is defined by a tuple

$$
\pi=\left(S, C, \Theta, \tau,\left(W_{c, \theta}\right)_{c \in C, \theta \in \Theta}, X, b_{C}, \succ_{S}, \tilde{\succsim}_{C}\right) .
$$

For instance, one may realistically consider the job market of young researchers in which student types are graduate, new doctorate and experienced doctorate. Each college $c$ proposes a set of possible wages $W_{c, \theta}$ to each type of student $\theta$. It is easy to see that typed weighted markets are a particular case of weighted markets, in which we require additional constraints on possible wages and the preferences of colleges.

For instance, in Fig. 1, there are two colleges $c_{1}$ and $c_{2}$, and two types of students: type $\theta_{1}$ which includes $s_{1}, s_{2}$ and $s_{3}$, and type $\theta_{2}$ which includes $s_{4}$ and $s_{5}$. Every student has a preference over his acceptable contracts. The possible wage between (for instance) college $c_{1}$ and students of type $\theta_{1}$ are wages set $W_{c_{1}, \theta_{1}}=\{3,2\}$. This is indeed a typed weighted market, because every college ranks higher his acceptable students of a higher type. For instance, concerning $c_{2}$, students $s_{3}$ and $s_{1}$ of type $\theta_{1}$, are ranked higher than $s_{4}$ and $s_{5}$, of type $\theta_{2}$. This typed weighted market amounts to a weighted market with possible contracts $X=$ $\left\{\left(s_{1}, c_{1}, 3\right),\left(s_{1}, c_{1}, 2\right),\left(s_{1}, c_{2}, 3\right),\left(s_{2}, c_{1}, 3\right),\left(s_{2}, c_{1}, 2\right),\left(s_{2}, c_{2}, 3\right),\left(s_{3}, c_{1}, 3\right),\left(s_{3}, c_{1}, 2\right),\left(s_{3}, c_{2}, 3\right)\right.$, $\left.\left(s_{4}, c_{1}, 1\right),\left(s_{4}, c_{2}, 1\right),\left(s_{5}, c_{1}, 1\right),\left(s_{5}, c_{2}, 1\right)\right\}$.

In our model, to choose an optimal subset of contracts, we need to know $\tilde{\succsim}_{c}$. For example, assume $s_{1} \succ_{c} s_{2} \succ_{c} s_{3}$ holds and $b_{c}=2$. When choosing an optimal subset within $\left\{\left(s_{1}, c, 2\right),\left(s_{2}, c, 1\right),\left(s_{3}, c, 1\right)\right\}$, we cannot tell whether college $c$ prefers $\left\{\left(s_{1}, c, 2\right)\right\}$ or

5. Students of a higher type can be considered unacceptable to a college, even if students of a lower type are considered acceptable. 


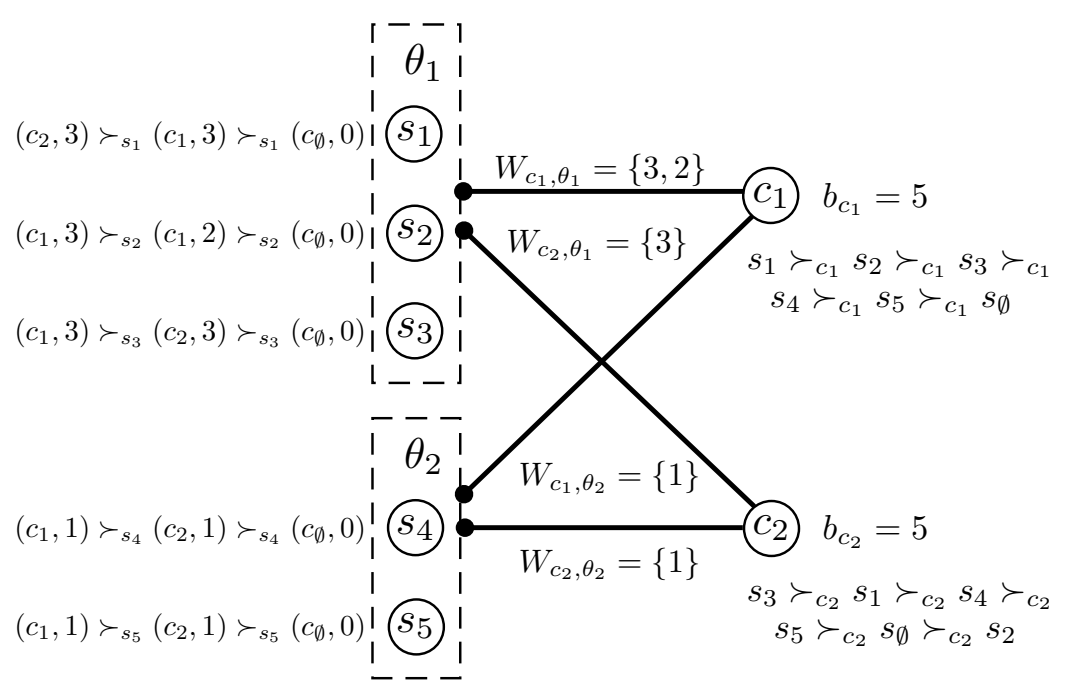

Figure 1: Example of typed-weighted market with two types of students.

$\left\{\left(s_{2}, c, 1\right),\left(s_{3}, c, 1\right)\right\}$ without $\tilde{\succsim}_{c}$. Obtaining $\tilde{\succsim}_{c}$ is difficult since it is a preference over $2^{n}$ sets, unless it can be concisely represented.

For instance, for each college $c$, weak preference $\tilde{\succsim}_{c}$ can be represented by an additively separable utility: utility function $u_{c}: S \rightarrow \mathbb{Z}$ that additively extends to sets $S^{\prime} \subseteq S$ of students by $u_{c}\left(S^{\prime}\right)=\sum_{s \in S^{\prime}} u_{c}(s)$. Hence, given two sets of students $S^{\prime}, S^{\prime \prime} \in 2^{S}$, preference $S^{\prime} \tilde{\succ}_{c} S^{\prime \prime}$ holds if and only if inequality $u_{c}\left(S^{\prime}\right)>u_{c}\left(S^{\prime \prime}\right)$ also holds, and indifference $S^{\prime} \tilde{\sim}_{c} S^{\prime \prime}$ holds if and only if one has equality $u_{c}\left(S^{\prime}\right)=u_{c}\left(S^{\prime \prime}\right)$. This defines weak preference $\tilde{\succsim}_{c}=$ $\tilde{\succ}_{c} \cup \tilde{\sim}_{c}$. The null-student has utility $u_{c}\left(s_{\emptyset}\right)=0$. An additively separable utility satisfies responsiveness. Also, it is an instance of a simple weighted market.

\subsection{Matching}

Given contract $x \in X$, let $\left(x_{S}, x_{C}, x_{W}\right)$ respectively denote the student, college, and wage that are linked by contract $x$. Given a subset of contracts $Y \subseteq X$, let us denote the set of contracts of student $s \in S$ as $Y_{s}=\left\{x \in Y \mid x_{S}=s\right\}$ and the set of contracts of college $c \in C$ as $Y_{c}=\left\{x \in Y \mid x_{C}=c\right\}$.

Definition 3. A matching is a subset of contracts $Y \subseteq X$ where each student $s$ goes to at most one college: $\left|Y_{s}\right| \leq 1 .^{6}$

Given matching $Y \subseteq X$, we abuse notation $Y$ in a natural functional manner as follows. Let $Y(s) \in(C \times W) \cup\left\{\left(c_{\emptyset}, 0\right)\right\}$ denote the college (or home $c_{\emptyset}$ ) to which student $s$ is assigned and the corresponding wage. Let $Y(c) \subseteq S$ denote the set of students assigned to college $c$. This functional notation maps agents to what they state their preferences on.

Definition 4. Contract $(s, c, w)$ is feasible if $(c, w) \succ_{s}\left(c_{\emptyset}, 0\right)$ and $s \succsim_{c} s_{\emptyset}$. Matching $Y$ is student-feasible for student $s$ if $Y_{s}$ is feasible. Matching $Y$ is college-feasible for college $c$ if

6. Students without contracts stay home. 
all students in $Y_{c}$ are feasible and if the sum of the wages is budget-feasible: $\sum_{x \in Y_{c}} x_{W} \leq b_{c}$. Feasible matching $Y \subseteq X$ is a matching that is student-feasible for each student and collegefeasible for each college.

Without loss of generality, we assume throughout the paper that for each contract $(c, s, w) \in X$, preference $s \succsim_{c} s_{\emptyset}$ holds.

\subsection{Stability}

A pairwise stable matching is immune to pairwise deviations by blocking pairs.

Definition 5. For matching $Y$, tuple $(s, c) \in S \times C$ is a blocking pair if there exists $w \in W$ and $R \subseteq Y_{c}$ such that $(s, c, w) \in X \backslash Y$ and the following conditions hold:

1. $(c, w) \succ_{s} Y(s)$,

2. $(Y(c) \backslash R(c)) \cup\{s\} \tilde{\succ}_{c} Y(c)$, and

3. $\sum_{x \in Y_{c} \backslash R} x_{W}+w \leq b_{c}$.

In other words, $(s, c)$ is a blocking pair if $s$ prefers $(c, w)$ over her current contract, $c$ is willing to reject the subset of its contracts $R$ to accept $s$, and it satisfies budget constraints.

Definition 6. Feasible matching $Y$ is pairwise stable if it does not admit any blocking pair.

In standard models, the "weight" of each student against the capacity limit is the same. Thus, to define a blocking pair, it is usually sufficient to consider replacing exactly one student. In our model, the "weight" of a student can be different; it is determined by its wage. Thus, it is more natural to assume that a college can replace multiple students to accept a student in a blocking pair. The meaning of a blocking pair is that the college and the student in the blocking pair have incentives to deviate. There is no good reason why the deviation of the college must be restricted to just replacing another student.

Similarly, a coalitionally stable matching is immune to coalitional deviations, since it does not admit any.

Definition 7. For matching $Y$, tuple $\left(S^{\prime}, c\right) \in 2^{S} \times C$ is a blocking coalition if there exists $w_{s} \in W$ for each $s \in S^{\prime}$ and $R \subseteq Y_{c}$ such that $\left(s, c, w_{s}\right) \in X \backslash Y$ and the following hold:

1. $\forall s \in S^{\prime},\left(c, w_{s}\right) \succ_{s} Y(s)$,

2. $(Y(c) \backslash R) \cup S^{\prime} \tilde{\succ}_{c} Y(c)$, and

3. $\sum_{x \in Y_{c} \backslash R} x_{W}+\sum_{s \in S^{\prime}} w_{s} \leq b_{c}$.

In other words, $\left(S^{\prime}, c\right)$ is a blocking coalition if each $s \in S^{\prime}$ prefers $\left(c, w_{s}\right)$ over her current contract, $c$ is willing to reject the subset of its contracts $R$ in order to accept $S^{\prime}$, and doing so satisfies its budget constraint.

Definition 8. Feasible matching $Y$ is coalitionally stable if it admits no blocking coalition. 
From the above definition, if $Y$ is coalitionally stable, it is also pairwise stable, but not vice versa. Furthermore, one may wonder why coalitional stability is not defined between a group of colleges and students (instead of one college and students). In fact, with regard to core stability, both concepts are equivalent. Colleges are indifferent to the other colleges in the same coalition (and students have preferences on colleges, not co-students). Therefore, a blocking coalition defined between a group of colleges and a group of students is the union of blocking coalitions with one college and many students. Consequently, since core stability asks whether there exists a blocking coalition, both concepts of blocking coalition (with one or many colleges) are equivalent with regard to core stability.

\section{The Complexity of Coalitional Stability in Weighted Markets}

In the field of computational complexity, a decision problem is modeled by an infinite set of instances and a question that maps each instance to yes or no. The answer is the desired output. In this section, we assume additively separable utilities for colleges, so that condition 2 in Definitions 5 and 7 are rewritten with sums. First, we observe that a coalitionally stable matching is not guaranteed to exist in every weighted market. This fundamental observation lets us introduce the coalitional stability in weighted market (CSWM) problem to decide whether a given weighted market admits (yes or no) a coalitionally stable matching.

For verifications in the CSWM problem, we then address the CSWM-Y problem to decide whether in a given weighted market, a given matching is coalitionally stable. We show that the CSWM-Y problem is coNP-complete. Hence, verification for CSWM does not seem polynomial-time tractable and CSWM is likely to fall outside of NP and coNP.

Ultimately, we show that (indeed) the CSWM problem is $\mathrm{NP}^{\mathrm{NP}}$-complete, even if the preferences of colleges on students are strict. Therefore, coalitional stability is a computationally very hard requirement in weighted markets.

\subsection{A Coalitionally Stable Matching Is Not Guaranteed to Exist}

Theorem 1. In simple weighted typed markets, coalitionally stable matchings may not exist.

Example 1. Consider this simple weighted market in which each possible contract is represented by a weighted edge.

$$
\begin{aligned}
& \left(c_{1}, 2\right) \succ_{s_{1}}\left(c_{2}, 2\right) \succ_{s_{1}}\left(c_{\emptyset}, 0\right) \\
& \left(c_{1}, 1\right) \succ_{s_{2}}\left(c_{2}, 1\right) \succ_{s_{2}}\left(c_{\emptyset}, 0\right) \\
& \left(c_{2}, 1\right) \succ_{s_{3}}\left(c_{1}, 1\right) \succ_{s_{3}}\left(c_{\emptyset}, 0\right)
\end{aligned}
$$

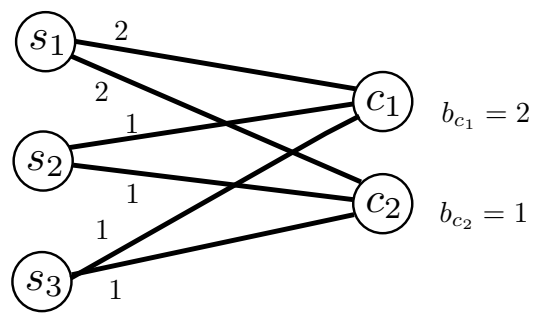

The possible contracts are $X=\left\{\left(s_{1}, c_{1}, 2\right),\left(s_{1}, c_{2}, 2\right),\left(s_{2}, c_{1}, 1\right),\left(s_{2}, c_{2}, 1\right),\left(s_{3}, c_{1}, 1\right),\left(s_{3}, c_{2}, 1\right)\right\}$, and the preference of each college $c$ is $s_{1} \succ_{c} s_{2} \succ_{c} s_{3} \succ_{c} s_{\emptyset}$ and extends to:

$$
\ldots \tilde{\succ}_{c}\left\{s_{2}, s_{3}\right\} \tilde{\succ}_{c}\left\{s_{1}\right\} \tilde{\succ}_{c}\left\{s_{2}\right\} \tilde{\succ}_{c}\left\{s_{3}\right\} \tilde{\succ}_{c} \emptyset \text {. }
$$


Specifying preference $\succ_{c}$ beyond $\left\{s_{2}, s_{3}\right\}$ is not useful, since further sets of students are not budget-feasible. Such a college preference could be obtained by additively separable utility $u_{c}\left(s_{1}\right)=4, \quad u_{c}\left(s_{2}\right)=3, \quad u_{c}\left(s_{3}\right)=2$.

This example can also be modeled as a typed weighted market, where $\Theta=\left\{\theta_{1}, \theta_{2}\right\}$, $\tau\left(s_{1}\right)=\theta_{1}, \tau\left(s_{2}\right)=\tau\left(s_{3}\right)=\theta_{2}$, and for every college $c$, one has: $W_{c, \theta_{1}}=\{2\}, W_{c, \theta_{2}}=\{1\}$.

Proof. We discuss all possible matchings $Y$ of Example 1, starting by $Y\left(c_{1}\right)$, which is either $\emptyset,\left\{s_{2}\right\},\left\{s_{3}\right\},\left\{s_{2}, s_{3}\right\}$ or $\left\{s_{1}\right\}$. Due to budget constraint, $s_{1}$ cannot be assigned to $c_{2}$.

Case 1: If $Y\left(c_{1}\right)=\emptyset$, then $\left(s_{1}, c_{1}\right)$ or $\left(s_{2}, c_{1}\right)$ blocks $Y$.

Case 2: If $Y\left(c_{1}\right)=\left\{s_{2}\right\}$ or $Y\left(c_{1}\right)=\left\{s_{3}\right\}$, then $\left(s_{1}, c_{1}\right)$ blocks $Y$.

Case 3: If $Y\left(c_{1}\right)=\left\{s_{2}, s_{3}\right\}$, then $\left(s_{3}, c_{2}\right)$ blocks $Y$.

Case 4: If $Y\left(c_{1}\right)=\left\{s_{1}\right\}$ and $Y\left(c_{2}\right) \neq\left\{s_{2}\right\}$, then $\left(s_{2}, c_{2}\right)$ blocks $Y$.

Case 5: If $Y\left(c_{1}\right)=\left\{s_{1}\right\}$ and $Y\left(c_{2}\right)=\left\{s_{2}\right\}$, then $\left(\left\{s_{2}, s_{3}\right\}, c_{1}\right)$ blocks $Y$.

Each matching is blocked by a coalition: Example 1 has no coalitionally stable matching.

\subsection{Reminders on Computational Complexity}

Class $P$ (polynomial-time) corresponds to the decision problems that can be answered in polynomial-time. Traditionally, we regard these problems as easy or tractable.

Class NP (non-deterministic polynomial-time) corresponds to the set of decision problems whose 'yes'-instances have a certificate verifiable in polynomial-time. For instance, consider the SUBSETSum problem: given a target $\alpha \in \mathbb{N}$ and a multiset $\mathcal{S}=\left\{w_{1}, \ldots, w_{n}\right\}$ of weights, the question asks whether there exists a subset of items $\mathcal{T} \subseteq \mathcal{S}$ that satisfies the constraint $\sum_{w \in \mathcal{T}} w=\alpha$ (hits the target). For 'yes'-instances, providing such a solution is an easy-to-check yes certificate, ${ }^{7}$ hence the SubSETSum problem is in NP.

Complementation consists in transposing the yes and no answers, e.g., the COSUBSETSum problem asks whether $\forall \mathcal{T} \subseteq \mathcal{S}, \sum_{w \in \mathcal{T}} w \neq \alpha$. The 'no'-instances are polynomial-time verifiable. This defines the problems of class coNP.

Problem SuBSETSUm is part of the most difficult problems of class NP, where a polynomialtime algorithm seems inexistent. Indeed, SubsetSum is NP-complete (Karp, 1972):

1. it belongs to NP,

2. it is NP-hard since every problem in NP reduces in polynomial time to SubSETSum. Hence, the existence of a polynomial-time algorithm for SUBSETSum would imply $\mathrm{P}=\mathrm{NP}$, which is widely believed to be false and argues for the intractability of SubSETSum. ${ }^{8}$

For some decision problems, neither yes nor no certification is polynomial-time tractable. In such cases, the problem falls outside of NP and coNP. Class $N P^{N P}$ corresponds $^{9}$ to the decision problems in which 'yes'-instances have proofs verifiable in polynomial time by using a constant-time NP-oracle. Class coNPNP is its complement. For instance, let us introduce the following new decision problems:

7. Guessing subset $\mathcal{T}$ is the non-deterministic part.

8. Similarly, one can show that a problem is coNP-complete by proving that it is in coNP and that it is the complement of an NP-hard problem, since NP and coNP are symmetric classes.

9. Class $\Sigma_{2}^{P}$ in the second level of the Polynomial Hierarchy. 


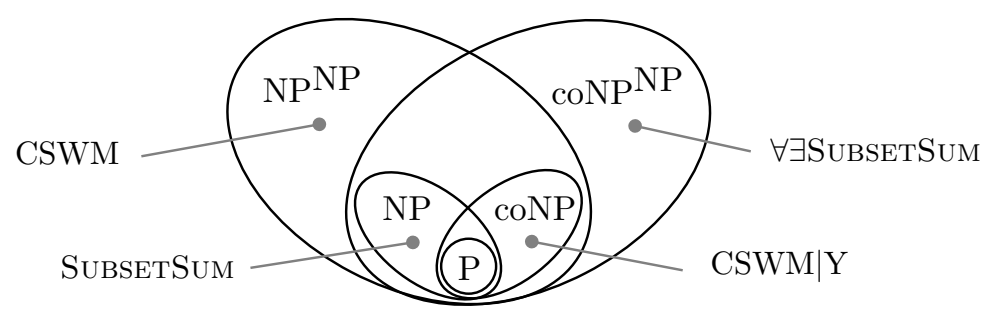

Figure 2: Inclusions of decision problem classes.

Definition 9. Given target $\alpha \in \mathbb{N}$ and two multi-sets of integers $\mathcal{S}^{\forall}$ and $\mathcal{S}^{\exists}$, the $\forall \exists$ SubSETSum problem asks whether

$$
\forall \mathcal{T}^{\forall} \subseteq \mathcal{S}^{\forall}, \quad \exists \mathcal{T}^{\exists} \subseteq \mathcal{S}^{\exists}, \quad \text { s.t. } \quad \sum_{w \in \mathcal{T}^{\forall}} w+\sum_{w \in \mathcal{T}^{\exists}} w=\alpha
$$

Conversely, the $\exists \forall$ SuBSETSum problem asks whether formula

$$
\exists \mathcal{T}^{\forall} \subseteq \mathcal{S}^{\forall}, \forall \mathcal{T}^{\exists} \subseteq \mathcal{S}^{\exists}, \sum_{w \in \mathcal{T}^{\forall}} w+\sum_{w \in \mathcal{T}^{\exists}} w \neq \alpha
$$

is true. The latter is simply the complement of the former.

The $\exists \forall$ SubsetSum problem lies in class NPNP. Indeed, by guessing the right set $\mathcal{T}^{\forall}$, one can use the NP-oracle to solve the remaining COSUBSETSum problem and verify the 'yes' answer. Similarly, $\forall \exists$ SubSetSum is in class coNP ${ }^{N P}$. Completeness is defined in a standard manner with polynomial-time reductions. Showing that problem $\forall \exists$ SubSETSum is coNP ${ }^{\mathrm{NP}}$-complete is a middle step in the proof below.

\subsection{Complexity of Verification}

We now address the complexity of a classical yes verification. The CSWM-Y problem, given weighted market $\pi=\left(S, C, W, X, b_{C}, \succ_{S}, \tilde{\succsim}_{C}\right)$ and feasible matching $Y$, asks whether $Y$ is (yes or no) coalitionally stable.

Theorem 2. The CSWM-Y problem is coNP-complete, even for a simple weighted typed market with only one college that has an additively separable utility.

Proof. First, the CSWM-Y problem is in coNP, since providing a blocking coalition $(T, c)$ is a no-certificate that can be verified in polynomial-time. Second, the complement of CSWM-Y (which answers 'yes' if there is a blocking coalition) is NP-hard, as we reduce SubSETSum to co-CSWM-Y.

Let set $\mathcal{S}=\left\{w_{1}, \ldots, w_{n}\right\}$ and target $\alpha \in \mathbb{N}$ be an instance of SubsetSum. We construct in polynomial-time the following CSWM-Y instance that addresses it. In this simple weighted market, there are students $S=\left\{s_{1}, \ldots, s_{n}, s_{\alpha}\right\}$ and one college $c$. College $c$ has budget $\alpha$. The wages and utilities are the same $w\left(c, s_{i}\right)=u_{c}\left(s_{i}\right)=w_{i}$ for $1 \leq i \leq n$ and 


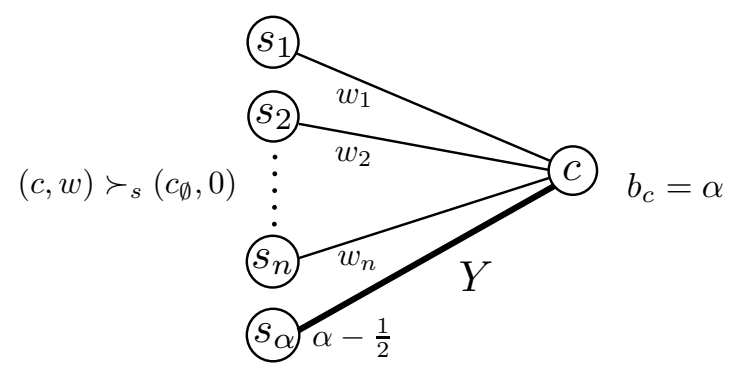

Figure 3: Reducing SubsetSum to co-CSWM-Y.

$w\left(c, s_{\alpha}\right)=u_{c}\left(s_{\alpha}\right)=\alpha-1 / 2$ for the last student. ${ }^{10}$ The preferences of students are to go to college $c$ rather than staying at home. The preference of the college is to maximize its utility, which here precisely corresponds to maximizing its budget consumption. In the given matching $Y=\left\{\left(s_{\alpha}, c, \alpha-1 / 2\right)\right\}$, student $s_{\alpha}$ goes to college $c$, and all other students go home. This reduction is depicted in Fig. 3. The college's budget consumption corresponds to its utility. Also, since wages and utilities correspond, this market is typed. It is straightforward that a blocking coalition exists if and only if a subset of items hits target $\alpha$.

If there is a subset $\mathcal{T} \subseteq \mathcal{S}$ which hits the target $\alpha$, then there is the corresponding blocking coalition $(T, c)$ which would improve the college's interest from $\alpha-1 / 2$ to $\alpha$. If no subset of items hits target $\alpha$, then no feasible coalition of students is better for college $c$ than $u_{c}\left(s_{\alpha}\right)=\alpha-1 / 2$.

\subsection{The Complexity of Coalitional Stability}

The previous subsection suggests that certification is hard, and hence that the problem CSWM falls outside of NP and coNP. Indeed, it is even harder than NP-complete.

Theorem 3. The CSWM problem is $N P^{N P}$-complete, even for a number of colleges in $O(1)$.

Proof, assuming Lemma 2 and 3. The CSWM problem is in $\mathrm{NP}^{\mathrm{NP}}$ since 'yes'-instances can be certified by this two step meta-algorithm.

1. Guess a coalitionally stable matching $Y$.

2. By using the NP-oracle on the corresponding CSWM-Y instance, prove that $Y$ is coalitionally stable.

For completeness in $\mathrm{NP}^{\mathrm{NP}}$, we equivalently show that problem coCSWM is coNP ${ }^{\mathrm{NP}}$ complete. The proof proceeds in two steps. First, we reduce the coNP ${ }^{\mathrm{NP}}$-complete problem $\forall \exists 3 \mathrm{CNF}$ to problem $\forall \exists$ SubsetSum (Lemma 2 ). Second, we reduce problem $\forall \exists$ SubsetSum to problem coCSWM (Lemma 3), achieving the proof.

Let $(\mathbb{B}=\{0,1\}, \vee, \wedge, \neg)$ denote the usual Boolean algebra. Given a set of variables $V$, an instantiation $I: V \rightarrow \mathbb{B}$ maps each variable $v \in V$ to a Boolean value $I(v) \in \mathbb{B}$. Given a Boolean variable $v$, the literals it induces are $\{v, \neg v\}$. A 3 -clause is the disjunction of 3

10. To have only integers, as in the model, one might multiply all the numbers by 2 and obtain a strategically equivalent market, or allow for half integers in the model. 
literals. A Boolean formula is in 3 conjunctive normal form (3CNF) if it is the conjunction of a set of 3-clauses.

Definition 10. An instance of the $\forall \exists 3 \mathrm{CNF}$ problem is defined by two sets of Boolean variables $V^{\forall}, V^{\exists}\left(V^{\forall} \cap V^{\exists}=\emptyset\right)$ and by a $3 \mathrm{CNF}$ formula $\phi$ defined as the conjunction of a set of 3-clauses $C$ on the literals induced by $V^{\forall} \cup V^{\exists}$. It asks if

$$
\forall I^{\forall}: V^{\forall} \rightarrow \mathbb{B}, \quad \exists I^{\exists}: V^{\exists} \rightarrow \mathbb{B}, \quad \bigwedge_{c \in C} c\left(I^{\forall}, I^{\exists}\right) .
$$

Example 2. Let $V^{\forall}=\left\{v_{1}, v_{2}\right\}, V^{\exists}=\left\{v_{3}, v_{4}\right\}$ and $\phi=$

$$
\underbrace{\left(v_{1} \vee \neg v_{2} \vee \neg v_{3}\right)}_{c_{1}} \wedge \underbrace{\left(\neg v_{1} \vee v_{3} \vee \neg v_{4}\right)}_{c_{2}} \wedge \underbrace{\left(v_{2} \vee v_{3} \vee v_{4}\right)}_{c_{3}}
$$

Does there, for every instantiation of $\left\{v_{1}, v_{2}\right\}$, exists an instantiation of $\left\{v_{3}, v_{4}\right\}$, such that formula $\phi$ is true?

Lemma 1. Problem $\forall \exists 3 \mathrm{CNF}$ is coNP ${ }^{N P}$-complete (Meyer \& Stockmeyer, 1972).

Problem $\forall \exists 3 \mathrm{CNF}$ is prototypical for the second level of the polynomial hierarchy, because it uses two groups of quantifiers.

Lemma 2. Problem $\forall \exists$ SubsetSum is coNP $P^{N P}$-complete.

Proof of Lemma 2. We encode a given instance of problem $\forall \exists 3 \mathrm{CNF}$ into the numerical weights and target of a $\forall \exists$ SUBSETSUm instance. It helps to represent the reduction as in Table 2 where each line represents a weight and each column is a component of the weight in a base $B$ large enough in some sense below. The idea is that the weights/lines will act as decision variables, and the components/columns will act as constraints on the "variables". For instance, in Table 2, we have $w_{v_{1}}=1 B^{6}+1 B^{2}$ and $w_{v_{3}}=1 B^{4}+1 B^{1}+1 B^{0}$. Each variable and each 3-clause indexes a column; so there are $\left|V^{\forall} \cup V^{\exists}\right|+|C|$ columns. To never have remainders in any addition of weights, the numbers are represented in a base $B$ which is large enough: each column can be seen as one constraint which has to precisely sum to the target's same column content, in order to satisfy $\sum_{w_{i} \in \mathcal{T}^{\forall}} w_{i}+\sum_{w_{j} \in \mathcal{T}^{\exists}} w_{j}=\alpha$ from Definition 9. It is largely sufficient to take $B=2\left(\left|V^{\forall} \cup V^{\exists}\right|+|C|\right)+1$, since it largely surpasses the number of lines.

Intuitively, the quantified Boolean variables and their instantiations are precisely modeled by the following $2\left|V^{\forall}\right|+2\left|V^{\exists}\right|$ weights and their quantifications. Two weights are associated to each variable $v$, one per induced literal: $w_{v}$ and $w_{\neg v}$. Both have their variablecolumns $v$ that equal 1 and the other variable-columns that equal 0 . Also, for column $v$, the target is set to 1 ; so that exactly one literal-weight per-variable will be in $\mathcal{T}^{\forall} \cup \mathcal{T}^{\exists}$. For universally quantified variables $v \in V^{\forall}$, exactly one weight (for instance $w_{v}$ ) goes in the universally quantified set of items $\mathcal{S}^{\forall}$ and the other (for instance $w_{\neg v}$ ) goes in the existentially quantified set of items $\mathcal{S}^{\exists}$, so that selecting a subset $\mathcal{T}^{\forall} \subseteq \mathcal{S}^{\forall}$ is equivalent to choosing an instantiation of $V^{\forall}$ and the same universal quantification is modeled. For existentially quantified variables $v \in V^{\exists}$, both weights go to the set of items $\mathcal{S}^{\exists}$. 


\begin{tabular}{c|c||cccc|ccc||c}
\multicolumn{2}{c|}{ Weights: } & $v_{1}$ & $v_{2}$ & $v_{3}$ & $v_{4}$ & $c_{1}$ & $c_{2}$ & $c_{3}$ & goes in: \\
\hline \multirow{4}{*}{$V^{\forall}$} & $w_{v_{1}}$ & 1 & 0 & 0 & 0 & 1 & 0 & 0 & $\mathcal{S}^{\forall}$ \\
& $w_{\neg v_{1}}$ & 1 & 0 & 0 & 0 & 0 & 1 & 0 & $\mathcal{S}^{\exists}$ \\
& $w_{v_{2}}$ & 0 & 1 & 0 & 0 & 0 & 0 & 1 & $\mathcal{S}^{\forall}$ \\
& $w_{\neg v_{2}}$ & 0 & 1 & 0 & 0 & 1 & 0 & 0 & $\mathcal{S}^{\exists}$ \\
\hline \multirow{5}{*}{$V^{\exists}$} & $w_{v_{3}}$ & 0 & 0 & 1 & 0 & 0 & 1 & 1 & $\mathcal{S}^{\exists}$ \\
& $w_{\neg v_{3}}$ & 0 & 0 & 1 & 0 & 1 & 0 & 0 & $\mathcal{S}^{\exists}$ \\
& $w_{v_{4}}$ & 0 & 0 & 0 & 1 & 0 & 0 & 1 & $\mathcal{S}^{\exists}$ \\
& $w_{\neg v_{4}}$ & 0 & 0 & 0 & 1 & 0 & 1 & 0 & $\mathcal{S}^{\exists}$ \\
\hline \multirow{5}{*}{ slack } & $w_{c_{1}}$ & 0 & 0 & 0 & 0 & 1 & 0 & 0 & $\mathcal{S}^{\exists}$ \\
& $w_{c_{1}^{\prime}}$ & 0 & 0 & 0 & 0 & 1 & 0 & 0 & $\mathcal{S}^{\exists}$ \\
& $w_{c_{2}}$ & 0 & 0 & 0 & 0 & 0 & 1 & 0 & $\mathcal{S}^{\exists}$ \\
& $w_{c_{2}^{\prime}}$ & 0 & 0 & 0 & 0 & 0 & 1 & 0 & $\mathcal{S}^{\exists}$ \\
& $w_{c_{3}}$ & 0 & 0 & 0 & 0 & 0 & 0 & 1 & $\mathcal{S}^{\exists}$ \\
& $w_{c_{3}^{\prime}}$ & 0 & 0 & 0 & 0 & 0 & 0 & 1 & $\mathcal{S}^{\exists}$ \\
\hline \multirow{6}{*}{} & $\alpha$ & 1 & 1 & 1 & 1 & 3 & 3 & 3 & Target
\end{tabular}

Table 2: Reducing Example 2 to $\forall \exists$ SubsetSum: each line represents a weight; last line is the target. Every column amounts to a constraint on the weights we pick.

For the clause columns, each clause that literal $v$ (or $\neg v$ ) makes true is set to 1 and the others to 0 . Then, in the column of clause $c$, the target would be that the clause is made true at least once. Note also that a clause cannot be made true more than 3 times. Consequently, we introduce slack-weights to reach target 3: for each clause $c$, we add 2 weights $w_{c}$ and $w_{c^{\prime}}$ with a 1 on clause-column $c$. By construction, the reduction is polynomial. Let us show that the $\forall \exists 3 \mathrm{CNF}$ instance is a 'yes' one if and only if this $\forall \exists$ SUBSETSUm instance is also a 'yes' one. The main idea is that a weight $w_{v_{i}}$ (respectively $w_{\neg v_{i}}$ ) is equivalent to an instantiation of $v_{i}$ to true (respectively false).

(yes $\Rightarrow$ yes) Assume that for every instantiation $I^{\forall}: V^{\forall} \rightarrow \mathbb{B}$ there exists an instantiation $\exists I^{\exists}: V^{\exists} \rightarrow \mathbb{B}$ such that $\bigwedge_{c \in C} c\left(I^{\forall}, I^{\exists}\right)$ (i.e. every clause is true), and let us show that in our construction, for every subset $\mathcal{T}^{\forall} \subseteq \mathcal{S}^{\forall}$, there exists a subset $\mathcal{T}^{\exists} \subseteq \mathcal{S}^{\exists}$, such that $\sum_{w \in \mathcal{T}^{\forall}} w+\sum_{w \in \mathcal{T}^{\exists}} w=\alpha$.

Let $\mathcal{T}^{\forall}$ be any subset of $\mathcal{S}^{\forall}$. Recall that every weight $w_{v_{i}}$ in $\mathcal{S}^{\forall}$ is equivalent to instantiating a variable $v_{i} \in V^{\forall}$ to true. We build a set $\mathcal{T}^{\exists}$ that satisfies $\sum_{w \in \mathcal{T}^{\forall}} w+\sum_{w \in \mathcal{T}^{\exists}} w=\alpha$ from instantiation $I^{\exists}$, which satisfies $\bigwedge_{c \in C} c\left(I^{\forall}, I^{\exists}\right)$. First, if $w_{v_{i}}$ is not in $\mathcal{T}^{\forall}$, we put $w_{\neg v_{i}}$ in $\mathcal{T}^{\exists}$; hence all column-constraints $v_{i} \in V^{\forall}$ are satisfied. Second, for every $v_{j} \in V^{\exists}$, if instantiation $I^{\exists}\left(v_{j}\right)$ is true, we put weight $w_{v_{j}}$ in $\mathcal{T}^{\exists}$, otherwise we put weight $w_{\neg v_{j}}$ in $\mathcal{T}^{\exists}$; hence all column-constraints $v_{j} \in V^{\exists}$ are satisfied. And more importantly, since $\bigwedge_{c \in C} c\left(I^{\forall}, I^{\exists}\right)$ is satisfied, every clause-column constraint $c_{i}$ is too (by using slack weights if necessary).

(yes $\Leftarrow$ yes) Assume that in our construction, for every subset $\mathcal{T}^{\forall} \subseteq \mathcal{S}^{\forall}$, there exists a subset $\mathcal{T}^{\exists} \subseteq \mathcal{S}^{\exists}$, such that $\sum_{w \in \mathcal{T}^{\forall}} w+\sum_{w \in \mathcal{T}^{\exists}} w=\alpha$, and let us show that for every instantiation $I^{\forall}: V^{\forall} \rightarrow \mathbb{B}$ there exists an instantiation $\exists I^{\exists}: V^{\exists} \rightarrow \mathbb{B}$ such that $\bigwedge_{c \in C} c\left(I^{\forall}, I^{\exists}\right)$ (i.e. every clause is true). 
Let $I^{\forall}: V^{\forall} \rightarrow \mathbb{B}$ be any instantiation of $V^{\forall}$ and let us build an instantiation $I^{\exists}: V^{\exists} \rightarrow \mathbb{B}$ that satisfies $\bigwedge_{c \in C} c\left(I^{\forall}, I^{\exists}\right)$. From instantiation $I^{\forall}$, our interest goes to $\mathcal{T}^{\forall}=\left\{w_{v_{i}} \in \mathcal{S}^{\forall} \mid\right.$ $I\left(v_{i}\right)=$ true $\}$. Then, the corresponding set $\mathcal{T}^{\exists}$ which satisfies $\sum_{w \in \mathcal{T}^{\forall}} w+\sum_{w \in \mathcal{T}} w=\alpha$ shows us how to build $I^{\exists}$, as follows: for every variable $v_{j} \in V^{\exists}$, instantiation $I^{\exists}\left(v_{j}\right)$ is set to true if and only if $w_{v_{j}}$ is in set $\mathcal{T}^{\exists}$. From constraint-columns $v_{i}$, it follows that it is indeed an instantiation; and from constraint-columns $c_{i}$, since we must hit a target three and there are only two slack-weights per clause, clause $c_{i}$ is satisfied by at least one variable.

Lemma 3. Problem $\forall \exists$ SubsetSum reduces to coCSWM.

Proof of Lemma 3. Let integer multisets $\mathcal{S}^{\forall}=\left\{\ldots, w_{i}, \ldots\right\}$ and $\mathcal{S}^{\exists}=\left\{\ldots, w_{j}, \ldots\right\}$, and integer target $\alpha \in \mathbb{N}$ define an instance of $\forall \exists$ SUBSETSum that we reduce to the following instance of coCSWM. Recall that problem coCSWM asks whether for all matchings there exists a blocking coalition.

Without loss of generality, we rule out the case in which $\sum_{w_{i} \in \mathcal{S}^{\forall}} w_{i}>\alpha$. We introduce a number $M$ that is large enough, e.g., $M=\sum_{w \in \mathcal{S}^{\forall} \cup \mathcal{S}^{\exists}} w$. From multisets $\mathcal{S}^{\forall}, \mathcal{S}^{\exists}$ of the $\forall \exists$ SubSETSum instance, we make two sets of students $S^{\forall}$ and $S^{\exists}$ in the coCSWM instance: for each item $w_{i} \in \mathcal{S}^{\forall}$, we introduce a student $s_{i} \in S^{\forall}$; and for each item $w_{j} \in \mathcal{S}^{\exists}$, we introduce a student $s_{j} \in S^{\exists}$. Then, we define three colleges: $c_{\forall \emptyset}, c_{\forall \exists}$ and $c_{\exists \emptyset}$. The budgets of the colleges are: $b_{c_{\forall \emptyset}}=M, b_{c_{\forall \exists}}=\alpha$ and $b_{c_{\exists \emptyset}}=M$. Finally, we append Example 1, by allowing student $s_{1}$ to go to college $c_{\forall \exists}$ with wage $w\left(s_{1}, c_{\forall \exists}\right)=1 / 2$ and giving to college $c_{\forall \exists}$ additional utility $u_{c_{\forall \exists}}\left(s_{1}\right)=1 / 2$. Crucially, if student $s_{1}$ is matched to college $c_{\forall \exists}$, then ${ }^{11}$ there is a coalitionally stable matching in Example 1; and otherwise, if $s_{1}$ is not matched to $c_{\forall \exists}$, then there is no coalitionally stable matching in Example 1, nor in the whole coCSWM instance, which is then a 'yes' instance.

For college $c_{\forall \emptyset}$, hiring a student from $S^{\forall}$ costs 0 and adds utility 0 . For college $c_{\exists \emptyset}$, hiring a student from $S^{\exists}$ costs 0 and adds utility 0 . Hence colleges $c_{\forall \emptyset}$ and $c_{\exists \emptyset}$ can hire any subset of students from $S^{\forall}$ (resp. $S^{\exists}$ ), but are indifferent to the sets of students that they receive. For college $c_{\forall \exists}$, hiring student $s_{i}$ from $S^{\forall} \operatorname{costs} w_{i}$ (the corresponding weight in the $\forall \exists$ SubsetSum instance) and adds utility $M$. Also, hiring student $s_{j}$ from $S^{\exists}$ costs $w_{j}$ and adds utility $w_{j}$. As a consequence, the preference of college $c_{\forall \exists}$ is lexicographically to:

1. Take all the students from $S^{\forall}$ who come,

2. Maximize budget consumption with students from $S^{\exists}$, while trying to hit budget $\alpha$.

3. If budget consumption does not hit $\alpha$, hire student $s_{1}$.

For every student $s_{i}$ in $S^{\forall}$, her preference $\left(c_{\forall \emptyset}, 0\right) \succ_{s_{i}}\left(c_{\forall \exists}, w_{i}\right) \succ_{s_{i}}\left(c_{\emptyset}, 0\right)$ means that her first choice is to go to college $c_{\forall \emptyset}$, while $c_{\forall \emptyset}$ is indifferent between hiring her or not. In a matching, let $T^{\forall}$ denote the subset of students from $S^{\forall}$ matched to $c_{\forall \exists}$, and let $S^{\forall} \backslash T^{\forall}$ denote those matched to college $c_{\forall \emptyset}$. Note that no student from $S^{\forall}$ may form a blocking coalition: First, students in $T^{\forall}$ will not provide a strict interest to college $c_{\forall \emptyset}$ by deviating to it. Second, students in $S^{\forall} \backslash T^{\forall}$ are not interested in deviating to $c_{\forall \exists}$.

For every student $s_{j}$ in $S^{\exists}$, her preference $\left(c_{\exists \exists}, w_{j}\right) \succ_{s_{j}}\left(c_{\exists \emptyset}, 0\right) \succ_{s_{j}}\left(c_{\emptyset}, 0\right)$ means that her first choice is to go to college $c_{\forall \exists}$, which enthusiastically welcomes her. Similarly, let

11. because $s_{1}$ prefers $\left(c_{\forall \exists}, 1 / 2\right)$ on top of his preference in Example 1 


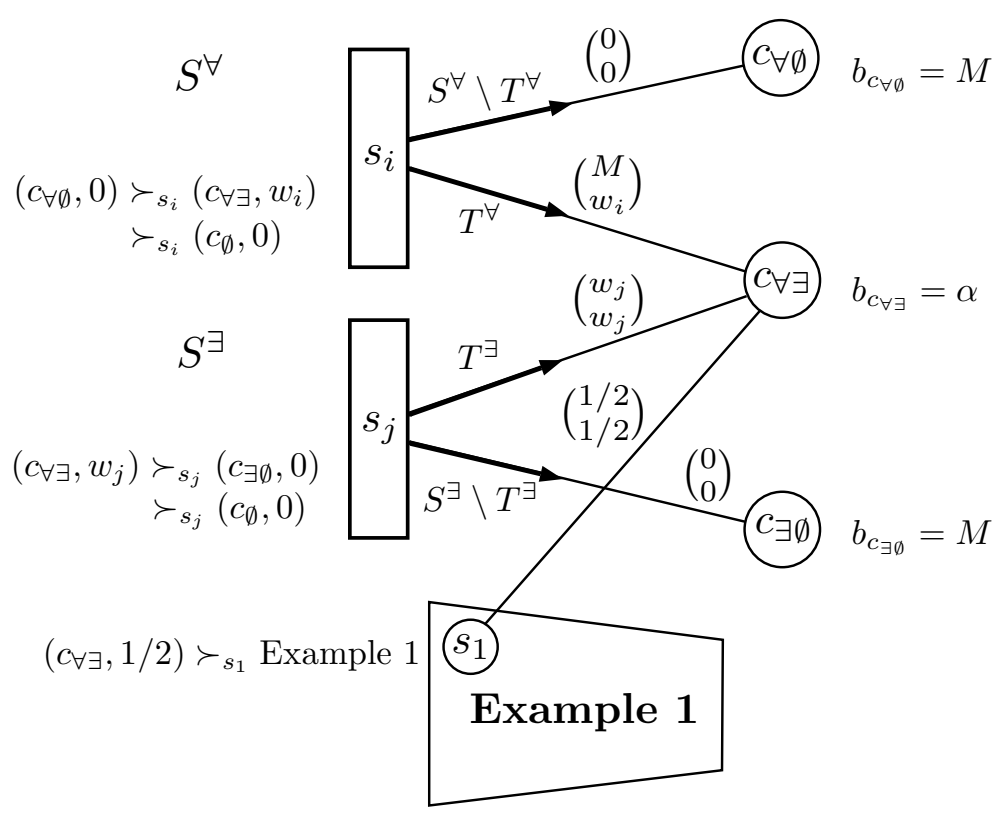

Figure 4: Reducing $\forall \exists$ SubsetSum to coCSWM.

$T^{\exists}$ denote the subset of students from $S^{\exists}$ matched to college $c_{\forall \exists}$, and let $S^{\exists} \backslash T^{\exists}$ denote those matched to $c_{\exists \emptyset}$.

(no $\Rightarrow$ no.) Assume that the $\forall \exists$ SUBSETSum instance is a 'no'-instance, which means

$$
\exists \mathcal{T}^{\forall} \subseteq \mathcal{S}^{\forall}, \quad \forall \mathcal{T}^{\exists} \subseteq \mathcal{S}^{\exists}, \quad \sum_{w_{i} \in \mathcal{T}^{\forall}} w_{i}+\sum_{w_{j} \in \mathcal{T}^{\exists}} w_{j} \neq \alpha
$$

and let us show that there exists a coalitionally stable matching. We construct this matching as follows. The set of students $T^{\forall}$ given by $\mathcal{T}^{\forall}$ in the above formula goes to college $c_{\forall \exists}$. Then, college $c_{\forall \exists}$ hires the subset of students $T^{\exists}$ that maximizes its budget consumption, but it does not hit target $\alpha$, because of the conditions on isomorphic sets $\mathcal{T}^{\exists}$ in the above formula. Finally, college $c_{\forall \exists}$ hires student $s_{1}$, and there is a coalitionally stable matching in Example 1. This matching is coalitionally stable: Since college $c_{\forall \emptyset}$ is universally indifferent, no blocking coalition can form with it. The same holds for $c_{\exists \emptyset}$. Since college $c_{\forall \exists}$ already maximizes budget consumption, no blocking coalition can form with it either. Finally, without $s_{1}$, Example 1 can be made stable.

(yes $\Rightarrow$ yes.) Assume that the $\forall \exists$ SubsetSum instance is a 'yes'-instance, which means

$$
\forall \mathcal{T}^{\forall} \subseteq \mathcal{S}^{\forall}, \quad \exists \mathcal{T}^{\exists} \subseteq \mathcal{S}^{\exists}, \quad \sum_{w_{i} \in \mathcal{T}^{\forall}} w_{i}+\sum_{w_{j} \in \mathcal{T}^{\exists}} w_{j}=\alpha
$$

Let us show that every matching admits a blocking coalition. Assume for the sake of contradiction that there exists a coalitionally stable matching. Then college $c_{\forall \exists}$ necessarily hired student $s_{1}$ and achieves at best budget consumption $\alpha-1 / 2$. However, college $c_{\forall \exists}$ could achieve budget consumption $\alpha$, since students from $S^{\exists}$ prefer a contract with $c_{\forall \exists}$ on top of everything. 
Indeed, let $T^{0}$ be the set of students corresponding to $\mathcal{T}^{\exists}$ in formula above and let $T \subseteq T^{0}$ those who are not yet matched with $c_{\forall \exists}$. Then $\left(T, c_{\forall \exists}\right)$ is a blocking coalition, which contradicts coalitional stability. Therefore, every matching admits a blocking coalition.

Theorem 1 extends to typed weighted markets, since both colleges rank students the same (hence one type per student proves it). Theorem 2 also extends to typed weighted markets, since there is only one college (again, one type per student). Concerning Theorem 3 , it is yet an open question.

\subsection{Extension to Strict Preferences}

Having college preferences that place strict orders on individual students, is often a fundamental requirement. However, Theorems 2 and 3 involve weak orders $\succsim_{c}$ for the preferences of colleges over individual students. In this subsection, we extend our complexity results to strict preferences $\succ_{c}$ over individual students and show the following:

Theorem 4 (On strict preferences for colleges over individual students).

(i) Theorem 2 holds, even if the college has a strict preference.

(ii) Lemma 2 holds, even if all the weights are distinct.

(iii) Lemma 3 holds, even if each college c has distinct utilities $\left(u_{c}(s) \mid s \in S\right)$.

(iv) Theorem 3 holds, even if each college has a strict preference.

We show all four points by extending the above proofs.

Proof of (i). Problem SubsetSum is still NP-complete when all the weights are different (Karp, 1972). Consequently, the same reduction as in Theorem 2 holds.

Proof of (ii). Lemma 2 reduces problem $\forall \exists 3$ CNF to problem $\forall \exists$ SUBSETSum as depicted in Table 2. In order to extend this reduction to weights all distinct, observe in Table 2 that the only weights being equal (which we do not want) are weights $w_{c}$ and $w_{c^{\prime}}$, for every clause $c$. Consequently, we add two columns $c^{(1)}$ and $c^{(2)}$ to differentiate weights $w_{c}$ and $w_{c^{\prime}}$ : we put a one on the first new column $c^{(1)}$ for $w_{c}$ and a one on the second new column $c^{(2)}$ for $w_{c^{\prime}}$, as depicted in Table 3. For these two new columns, the target is one. Also, to retain the freedom to either pick or refuse weights $w_{c}$ and $w_{c^{\prime}}$, we add two new slack weights (one per new column) with one 1 on the new column. For every clause $c$, we add two such new columns and the corresponding two new slack variables. The reduction still works and all the weights are different.

\begin{tabular}{|c|c|c|c|c|c|c|}
\hline \multicolumn{2}{|c|}{ Weights: } & (variables) & $c$ & $c^{(1)}$ & $c^{(2)}$ & goes in: \\
\hline \multicolumn{7}{|c|}{$\vdots$} \\
\hline \multirow[t]{2}{*}{ slack } & $w_{c}$ & $0 \ldots$ & 1 & 1 & 0 & $\mathcal{S}^{\exists}$ \\
\hline & $w_{c^{\prime}}$ & $0 \ldots$ & 1 & 0 & 1 & $\mathcal{S}^{\exists}$ \\
\hline \multirow[t]{3}{*}{ new slack } & $w_{c^{(1)}}$ & $0 \ldots$ & 0 & 1 & 0 & $\mathcal{S}^{\exists}$ \\
\hline & $w_{c^{(2)}}$ & $0 \ldots$ & 0 & 0 & 1 & $\mathcal{S}^{\exists}$ \\
\hline & $\alpha$ & $\ldots$ & 3 & 1 & 1 & Target \\
\hline
\end{tabular}

Table 3: Extension of Lemma 2's proof: zoom on weights $w_{c}$ and $w_{c^{\prime}}$. 
Proof of (iii). Here, we extend the proof of Theorem 3 to having for each college $c$ distinct utilities $u_{c}(s)$ on every feasible student $s$ (in order to get strict preferences for each college). In the former reduction which is depicted in Figure 4, observe that colleges $c_{\forall \emptyset}$ and $c_{\exists \emptyset}$ have identical utilities $u_{c_{\forall \emptyset}} \equiv 0$ and $u_{c_{\exists \emptyset}} \equiv 0$. Also, observe that for college $c_{\forall \exists}$, utilities $M$ from students in $S^{\forall}$ are identical. In this extension, we introduce distinct utilities as follows.

To be distinct, utilities $M$ are modified to $M$ plus Borda scores. That is, college $c_{\forall \exists}$ values students $s_{i} \in S^{\forall}$ by $u_{c \ni \exists}\left(s_{i}\right)=M+i$. This does not change the strategical interactions of college $c_{\forall \exists}$ with the students in $S^{\forall}$, or the reduction's validity.

Assume that the indices $i$ of students $s_{i}$ in $S^{\forall}$ start from zero; and recall that in the binary representation of $2^{i}$ there are only zeros but a one in the $i$ th position (starting from zero). For college $c_{\forall \emptyset}$, the utility and cost of each student $s_{i}$ in $S^{\forall}$ become $2^{2 i}+2^{2 i+1}$ (that is ones only in position $2 i$ and $2 i+1)$ and we modify the budget to $b_{c_{\forall \emptyset}}=\sum_{s_{i} \in S^{\forall}}\left(2^{2 i}+2^{2 i+1}\right)$. Also, for each student $s_{i}$ in $S^{\forall}$, we introduce two new students $s_{i}^{\prime}$ and $s_{i}^{\prime \prime}$, whose utility and cost are $2^{2 i}$ for $s_{i}^{\prime}$ and $2^{2 i+1}$ for $s_{i}^{\prime \prime}$; so that college $c_{\forall \emptyset}$ is indifferent between hiring student $s_{i}$ or students $s_{i}^{\prime}$ and $s_{i}^{\prime \prime}$ (as long as budget $b_{c_{\forall \emptyset}}$ is entirely consumed). Also, we apply the same extension for college $c_{\exists \emptyset}$. Letting colleges $c_{\forall \emptyset}$ and $c_{\exists \emptyset}$ hit there maximal budget consumption, the same proof holds.

Proof of (iv). Combining Theorem 4, parts (ii) and (iii), one obtains Claim (iv).

\subsection{On Blocking Coalitions with Bounded Size}

In this subsection, we constrain the size of blocking coalitions to at most a fixed number $\sigma$ of students (and one college). For instance, this can be the consequence of limited communications between students and colleges before deviating. Let $\sigma$-CSWM be the new corresponding problem on existence of a coalitionally stable matching. A coalitionally stable matching still isn't guaranteed to exist since Example 1 only involves coalitions of up to two students. Below, we show that $\sigma$-CSWM is NP-hard for $\sigma=2$ (even in typed markets), which closes the gap with pairwise stability $(\sigma=1)$.

Theorem 5. If blocking coalitions are constrained to at most two students, then the corresponding 2-CSWM problem is strongly NP-hard, even in simple weighted typed markets.

Proof. Since Example 1 still holds, the $\sigma$-CSWM problem remains a decision problem. For strong hardness, let any instance of Numerical Matching with Target Sums (NMTS) be defined by two disjoint sets $\mathcal{S}$ and $\mathcal{T}$, each containing $m$ elements, a size $v(a) \in \mathbb{N}_{>0}$ for each element $a \in \mathcal{S} \cup \mathcal{T}$, and a target vector $\left(B_{1}, B_{2}, \ldots, B_{m}\right)$ with $m$ positive integer entries. The problem asks whether $\mathcal{S} \cup \mathcal{T}$ can be partitioned into $m$ disjoint subsets $A_{1}, \ldots, A_{m}$, each $A_{i}$ containing exactly one element from each $\mathcal{S}$ and $\mathcal{T}$ (that is: $\left|A_{i} \cap \mathcal{S}\right|=\left|A_{i} \cap \mathcal{T}\right|=1$ ), such that for $1 \leq i \leq m, \sum_{a \in A_{i}} v(a)=B_{i}$ holds.

From this instance, we construct in polynomial-time the following instance of $\sigma$-CSWM with $\sigma=2$. Let $M=\sum_{i=1}^{m} B_{i}$ be a number larger than any other. There are two sets of students $S$ and resp. $T$ in correspondence with $\mathcal{S}$ and resp. $\mathcal{T}$; hence a total of $2 m$ students. There are $m$ colleges, in correspondence with vector $\left(B_{1}, \ldots, B_{m}\right)$. Each student has preference $c_{1} \succ c_{2} \succ \ldots \succ c_{m} \succ c_{\emptyset}$. Each college $c_{i}$ has budget $M^{2}+M+B_{i}$. Also, college $c_{i}$, for any student $a \in S$ has additive utility and wage $u_{c_{i}}(a)=w\left(c_{i}, a\right)=M^{2}+v(a)$, but for any student $b \in T$ has additive utility and wage $u_{c_{i}}(b)=w\left(c_{i}, b\right)=M+v(b)$. Since 
utilities and wages are the same, each college wants to maximize its budget consumption. Finally, we also insert Example 1. We also introduce one student $s_{\pi}$ who prefers to be with colleges $c_{1}, \ldots, c_{m}$ rather than the first college of Example 1, which in turn would hire student $s_{\pi}$ over anyone else, for wage 2 . Colleges $c_{1}, \ldots, c_{m}$ would hire student $s_{\pi}$ for wage and utility $u_{c_{i}}\left(s_{\pi}\right)=w\left(c_{i}, s_{\pi}\right)=1 / 2$. This is a typed market because wages and utilities are the same.

(Yes $\Rightarrow$ Yes) Assume the NMTS instance has a solution $A_{1}, \ldots, A_{m}$, and let us construct a coalitionally stable matching. For each $A_{i}=\{a, b\}$, we match the two corresponding students to college $c_{i}$, who pays $M^{2}+v(a)+M+v(b)=M^{2}+M+B_{i}$, hits his budget $B_{i}$ and is not interested in any deviation. Then, student $s_{\pi}$ is hired by the first college of Example 1. Consequently, the remainder of Example 1 is stable.

(Yes $\Leftarrow$ Yes) Assume that there exists a coalitionally stable matching $Y$, and let us construct a solution to the NMTS instance. The only way for matching $Y$ to be stable, is that Example 1 is disabled by its first college hiring student $s_{\pi}$. Then, every college $c_{i}$ did hit its budget consumption $B_{i}$. (Otherwise, student $s_{\pi}$ going to a college that still has some money in its budget would be a blocking coalition.) Because every college did hit budget consumption, exactly one student $a$ from set $S$ goes in every college. (Otherwise, some college would be missing value/budget-consumption $M^{2}$.) Then, for similar reasons, also exactly one student $b$ from set $T$ goes in every college. (Otherwise, some college would be missing value/budget-consumption $M$.) And since $M^{2}+v(a)+M+v(b)=M^{2}+M+B_{i}$ implies $v(a)+v(b)=B_{i}$, it follows that $A_{1} \equiv Y\left(c_{1}\right), \ldots, A_{m} \equiv Y\left(c_{m}\right)$ is a solution to the NMTS instance. To conclude, since NMTS is strongly NP-hard, so is problem $\sigma$-CSWM.

\section{Mechanism Design in Typed Weighted Markets}

The previous section suggests that requiring coalitional stability is hopeless, even in typed markets. In this section, we discuss a strategy-proof and pairwise stable mechanism for typed weighted markets called the sequential deferred acceptance (SDA) mechanism. When each college has a strict preference over individual students, SDA also satisfies a property

called constrained Pareto efficiency; SDA's outcome is Pareto efficient among pairwise stable outcomes.

\subsection{Pairwise Stable Matching Might Not Exist in General}

We first show that a pairwise stable matching is not guaranteed to exist in general. We utilize the following example.

Example 3. Consider a simple weighted market with three students $s_{1}, s_{2}$, and $s_{3}$, and two colleges $c_{1}$ and $c_{2}$, whose budgets are 2 and 1, respectively. Possible contracts are $X=\left\{\left(s_{1}, c_{1}, 2\right),\left(s_{2}, c_{1}, 1\right),\left(s_{2}, c_{2}, 1\right),\left(s_{3}, c_{1}, 1\right),\left(s_{3}, c_{2}, 1\right)\right\}$. The preference of $c_{1}$ is:

$$
\left\{s_{2}, s_{3}\right\} \tilde{\succ}_{c_{1}}\left\{s_{2}\right\} \tilde{\succ}_{c_{1}}\left\{s_{1}\right\} \tilde{\succ}_{c_{1}}\left\{s_{3}\right\} \tilde{\succ}_{c_{1}} \emptyset .
$$

Such a college preference can be obtained by additively separable utility $u_{c_{1}}\left(s_{1}\right)=3$, $u_{c_{1}}\left(s_{2}\right)=4, u_{c_{1}}\left(s_{3}\right)=2$. The preference of $c_{2}$ is:

$$
\left\{s_{3}\right\} \tilde{\succ}_{c_{2}}\left\{s_{2}\right\} \tilde{\succ}_{c_{2}} \emptyset .
$$


Here, we show the preferences only on feasible contracts. The preference of $s_{1}, s_{2}, s_{3}$ are:

$$
\begin{array}{ll}
s_{1}: & \left(c_{1}, 2\right) \succ_{s_{1}}\left(c_{\emptyset}, 0\right), \\
s_{2}: & \left(c_{2}, 1\right) \succ_{s_{2}}\left(c_{1}, 1\right) \succ_{s_{2}}\left(c_{\emptyset}, 0\right) \\
s_{3}: & \left(c_{1}, 1\right) \succ_{s_{3}}\left(c_{2}, 1\right) \succ_{s_{3}}\left(c_{\emptyset}, 0\right)
\end{array}
$$

This example is depicted in the following figure:

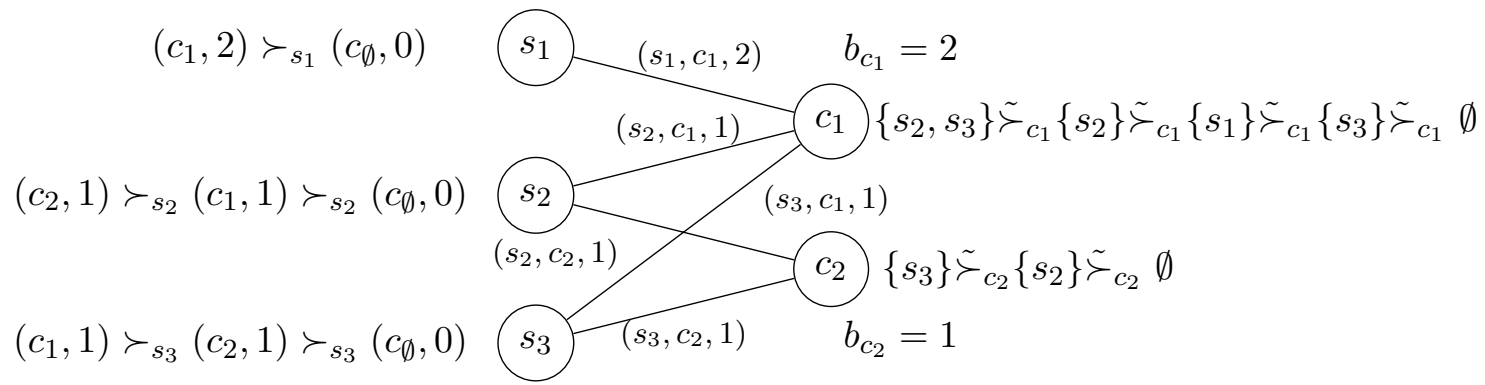

Theorem 6. There exists a case in which no pairwise stable matching exists.

Proof. In Example 3, due to budget constraints, $s_{1}$ cannot be matched to $c_{2}$. Thus, $s_{1}$ is either (a) matched to $c_{1}$, or (b) not matched to any college (i.e., she stays home).

First, let us examine case (a). Due to budget constraints, only $s_{1}$ is matched to $c_{1}$, and at most one student can be matched to $c_{2}$. Within the remaining students, $s_{3}$, who is the most preferred student for $c_{2}$, must be matched to $c_{2}$; otherwise, $\left(s_{3}, c_{2}\right)$ will be a blocking pair. Thus, $s_{2}$ stays home. However, then $\left(s_{2}, c_{1}\right)$ will be a blocking pair.

In case (b), both $s_{2}$ and $s_{3}$ must be matched to some college; otherwise, the unmatched student and $c_{1}$ will be a blocking pair (since $c_{1}$ can accept both students). Furthermore, $s_{3}$, who prefers $c_{1}$ over $c_{2}$, must be matched to $c_{1}$; otherwise, $\left(s_{3}, c_{1}\right)$ will be a blocking pair. Thus, there are two cases: (i) both are matched to $c_{1}$, or (ii) $s_{2}$ is matched to $c_{2}$, and $s_{3}$ is matched to $c_{1}$. In case (i), $\left(s_{2}, c_{2}\right)$ will be a blocking pair. In case (ii), $\left(s_{1}, c_{1}\right)$ will be a blocking pair. Since every possible matching admits a blocking pair, there is no pairwise stable matching in Example 3.

This result highlights the difference between our model and the model used in (Abizada, 2016), in which a pairwise-stable matching is guaranteed to exist. In our model, we allow different types of students to be in a market. In Example 3, we can assume $s_{2}$ and $s_{3}$ have the same type, while $s_{1}$ has a different type, thus $s_{1}$ should be hired by a different wage.

Note that this example cannot be modeled as a typed weighted market, since $c_{1}$ prefers $s_{2}$ over $s_{1}$, while the wage for $s_{1}$ is larger than that for $s_{2}$. Theorem 1 and Theorem 6 are disjoint, neither implies the other. Theorem 1 holds even in typed markets, which Theorem 6 precisely does not assume. As we show later in this paper, for a typed weighted market, we can guarantee that a pairwise stable matching always exists.

\subsection{Strategy-Proof Mechanism for Typed Weighted Markets}

Let us formally define a mechanism and strategy-proofness. Mechanism $\varphi$ is a function that takes a profile of the preferences of students $\succ_{S}$ as input and returns matching $Y$. 
Let $\succ_{S \backslash\{s\}}$ denote a profile of the preferences of the students except $s$, and let $\left(\succ_{s}, \succ_{S \backslash\{s\}}\right)$ denote a profile of the preferences of all the students, where $s$ 's preference is $\succ_{s}$ and the profile of the preferences of the other students is $\succ_{S \backslash\{s\}}$.

Definition 11. Mechanism $\varphi$ is strategy-proof for students if it holds that $Y(s) \succ_{s} Y^{\prime}(s)$ or $Y(s)=Y^{\prime}(s)$ for every $s, \succ_{s}, \succ_{s}^{\prime}$ and $\succ_{S \backslash\{s\}}$, where $Y=\varphi\left(\left(\succ_{s}, \succ_{S \backslash\{s\}}\right)\right)$ and $Y^{\prime}=\varphi\left(\left(\succ_{s}^{\prime}\right.\right.$

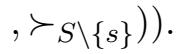

Definition 12. For two feasible matchings $Y$ and $Y^{\prime}$, matching $Y$ Pareto dominates matching $Y^{\prime}$ if (i) $\forall s \in S, Y(s) \succ_{s} Y^{\prime}(s)$ or $Y^{\prime}(s)=Y(s)$ holds, and (ii) $\exists s \in S$, s.t. $Y(s) \succ_{s} Y^{\prime}(s)$. Matching $Y$ is constrained Pareto efficient if $Y$ is pairwise stable and no other pairwise stable matching Pareto dominates $Y$. Mechanism $\varphi$ is constrained Pareto efficient if it always obtains a constrained Pareto efficient matching.

The SDA mechanism sequentially applies the (student-proposing) deferred acceptance mechanism (DA) (Gale \& Shapley, 1962), from highest type $\theta_{1}$ to lowest type $\theta_{k}$. The DA mechanism exploits the following crafted choice functions.

Definition 13 (Choice function of students). For each student $s$, her choice function $C h_{s}$ maps any subset of contracts $X^{\prime} \subseteq X$ to contract $\{x\}$, which is the most preferred contract in $X_{s}^{\prime}$ based on $\succ_{s}$ if one exists; otherwise $\emptyset$ if no feasible contract exists. The choice function of set of students $\hat{S}$, denoted as $C h_{\hat{S}}$, is defined as $C h_{\hat{S}}\left(X^{\prime}\right)=\bigcup_{s \in \hat{S}} C h_{s}\left(X^{\prime}\right)$, i.e., the union of choice functions of $\hat{S}$.

Definition 14 (Choice function of colleges). For every college $c$, choice function $C h_{c}\left(X^{\prime}\right)$ is defined as follows:

1. $Z \leftarrow \emptyset, Y \leftarrow X_{c}^{\prime}$.

2. Repeat the following procedure: If $Y=\emptyset$, return $Z$. Otherwise, remove $(s, c, w) \in Y$ with the highest priority ranking from $Y$, s.t. $s \succ_{c} s_{\emptyset}$, based on preference $\succsim_{c}$ on students (ties are broken in some deterministic way, e.g., based on the alphabetical order of the student identifiers). If $\sum_{x \in Z} x_{W}+w \leq b_{c}$, add $(s, c, w)$ to $Z$.

The choice function of all colleges is defined as $C h_{C}\left(X^{\prime}\right)=\bigcup_{c \in C} C h_{c}\left(X^{\prime}\right)$.

Note that the choice function for each college $c$ is crafted such that it does not exactly reflect $\tilde{\succsim}_{c}$. Actually, it is defined based on preference $\succsim_{c}$ over individual students. This fact is considered an advantage, since as discussed in Section 2 , obtaining $\tilde{\succsim}_{c}$ is difficult in general. As we show, we can guarantee strategy-proofness for students and pairwise stability using choice functions defined this way.

We use the following (student-proposing) DA as a component of our SDA mechanism. For a given set of students $\hat{S}, \mathrm{DA}$ is defined as follows, where $X_{\hat{S}}=\bigcup_{s \in \hat{S}} X_{s}$.

Definition 15 (Deferred Acceptance mechanism (DA)).

1. $R e \leftarrow \emptyset$.

2. $Y \leftarrow C h_{\hat{S}}\left(X_{\hat{S}} \backslash R e\right), Z \leftarrow C h_{C}(Y)$.

3. If $Y=Z$, then return $Y$, otherwise:

$R e \leftarrow R e \cup(Y \backslash Z)$, go to Step 2.

Here $R e$ represents the set of rejected contracts, $Y$ represents the contracts proposed by students $\hat{S}$ within $X_{\hat{S}} \backslash R e$, and $Z$ represents the contracts in $Y$ accepted by colleges. Thus, $Y \backslash Z$ represents a set of newly rejected contracts. 
Mechanism 1 (Sequential Deferred Acceptance)

Let $Y \leftarrow \emptyset$ and $i \leftarrow 1$.

Round $i$ :

1. Let $\hat{S} \leftarrow\left\{s \in S \mid \tau(s)=\theta_{i}\right\}$, i.e., the set of all type $\theta_{i}$ students, and run the DA.

2. Let $Y^{i}$ be the obtained matching. $Y \leftarrow Y \cup Y^{i}$.

3. If $i=k$ then return $Y$, otherwise:

$\forall c \in C, \quad b_{c} \leftarrow b_{c}-\sum_{x \in Y_{c}^{i}} x_{W}$

$i \leftarrow i+1 ;$ Go to Round $i$.

SDA is defined in Mechanism 1. It repeatedly applies DA for each type from $\theta_{1}$ to $\theta_{k}$.

Example 4. Let us describe the execution of SDA on the market illustrated in Fig. 1.

Round 1. We run DA for $\hat{S}=\left\{s_{1}, s_{2}, s_{3}\right\}$, i.e., all type $\theta_{1}$ students, under original budgets $b_{C}=(5,5)$. The iterations in DA are as follows:

1. $Y=\left\{\left(s_{1}, c_{2}, 3\right),\left(s_{2}, c_{1}, 3\right),\left(s_{3}, c_{1}, 3\right)\right\}$ and $Z=\left\{\left(s_{1}, c_{2}, 3\right),\left(s_{2}, c_{1}, 3\right)\right\} ; c_{1}$ rejects $\left(s_{3}, c_{1}, 3\right)$ because $s_{2} \succ_{c_{1}} s_{3}$ and its budget is 5 .

2. $Y=\left\{\left(s_{1}, c_{2}, 3\right),\left(s_{2}, c_{1}, 3\right),\left(s_{3}, c_{2}, 3\right)\right\}$ and $Z=\left\{\left(s_{2}, c_{1}, 3\right),\left(s_{3}, c_{2}, 3\right)\right\} ; c_{2}$ rejects $\left(s_{1}, c_{2}, 3\right)$ because $s_{3} \succ_{c_{2}} s_{1}$ and its budget is 5 .

3. $Y=\left\{\left(s_{1}, c_{1}, 3\right),\left(s_{2}, c_{1}, 3\right),\left(s_{3}, c_{2}, 3\right)\right\}$ and $Z=\left\{\left(s_{1}, c_{1}, 3\right),\left(s_{3}, c_{2}, 3\right)\right\} ; c_{1}$ rejects $\left(s_{2}, c_{1}, 3\right)$ because $s_{1} \succ_{c_{1}} s_{2}$ and its budget is 5 .

4. $Y=Z=\left\{\left(s_{1}, c_{1}, 3\right),\left(s_{2}, c_{1}, 2\right),\left(s_{3}, c_{2}, 3\right)\right\}$. All colleges satisfy their budget constraints. Therefore, we obtain $Y^{1}=\left\{\left(s_{1}, c_{1}, 3\right),\left(s_{2}, c_{1}, 2\right),\left(s_{3}, c_{2}, 3\right)\right\}$.

Round 2. We run DA for $\hat{S}=\left\{s_{4}, s_{5}\right\}$ with the remaining budget, i.e., $b_{c_{1}}=5-5=0$ and $b_{c_{2}}=5-3=2$. The iterations in DA are as follows:

1. $Y=\left\{\left(s_{4}, c_{1}, 1\right),\left(s_{5}, c_{1}, 1\right)\right\}$ and $Z=\emptyset$, because $c_{1}$ has no budget to accept any student.

2. $Y=Z=\left\{\left(s_{4}, c_{2}, 1\right),\left(s_{5}, c_{2}, 1\right)\right\}$. All colleges satisfy their budget constraints. Therefore, we obtain $Y^{2}=\left\{\left(s_{4}, c_{2}, 1\right),\left(s_{5}, c_{2}, 1\right)\right\}$.

To conclude, SDA returns the following matching:

$$
Y^{1} \cup Y^{2}=\left\{\left(s_{1}, c_{1}, 3\right),\left(s_{2}, c_{1}, 2\right),\left(s_{3}, c_{2}, 3\right),\left(s_{4}, c_{2}, 1\right),\left(s_{5}, c_{2}, 1\right)\right\} .
$$

Abizada (2016) presented a strategy-proof mechanism that works for a different model as described in Section 1, where each college $c$ has its budget limit $b_{c}$ and maximum wage $m_{c}$, and all students are the same type $\theta$. The mechanism proposed by Abizada (2016) can be considered as a special case of our component DA mechanism, where possible wages $W_{c, \theta}$ is restricted to $\left\{w_{1}^{c}, w_{2}^{c}, 0\right\}$ where $w_{1}^{c}=m_{c}, w_{2}^{c}=b_{c} \bmod m_{c}$.

\subsection{The Pairwise Stability of SDA}

In the following, we show that mechanism SDA outputs a pairwise stable matching. 
Theorem 7. SDA always returns a pairwise stable matching.

To prove this theorem, we use the following lemmas.

Lemma 4. Let $s \in S, S^{\prime} \subseteq S \backslash\{s\}$ s.t. $s^{\prime} \succsim_{c} s_{\emptyset}$ holds for all $s^{\prime} \in S^{\prime} \cup\{s\}$. Assume there exists $S^{\prime \prime} \subseteq S^{\prime}$ s.t. $S^{\prime} \backslash S^{\prime \prime} \cup\{s\} \tilde{\succ}_{c} S^{\prime}$ holds. Then $s \succ_{c} s^{\prime}$ holds for all $s^{\prime} \in S^{\prime \prime}$.

In other words, if college $c$, which currently has $S^{\prime}$, prefers adding $s$ by removing $S^{\prime \prime}$, then $c$ prefers $s$ over any student $s^{\prime} \in S^{\prime \prime}$. This is intuitively natural; if $s$ can defeat coalition $S^{\prime \prime}$, she can also defeat each individual in it. We formally prove this from the fact that $\tilde{\succsim}_{c}$ is responsive.

Proof of Lemma 4. Assume by way of contradiction that there exists $\hat{s} \in S^{\prime \prime}$ such that $\hat{s} \succsim_{c} s$ holds. Since we assume $\hat{s} \succsim_{c} s$ holds, from responsiveness, when we add either $\hat{s}$ or $s$ to $S^{\prime} \backslash\{\hat{s}\}$, we have $S^{\prime} \tilde{\succsim}_{c} S^{\prime} \backslash\{\hat{s}\} \cup\{s\}$. From the assumption, $s^{\prime} \succsim_{c} s_{\emptyset}$ holds for all $s^{\prime} \in S^{\prime} \cup\{s\}$. Thus, from responsiveness, by adding students in $S^{\prime \prime} \backslash\{\hat{s}\}$ one by one to $S^{\prime} \backslash S^{\prime \prime} \cup\{s\}$, we have $S^{\prime} \backslash\{\hat{s}\} \cup\{s\} \tilde{\succsim}_{c} S^{\prime} \backslash S^{\prime \prime} \cup\{s\}$. From these facts, we obtain $S^{\prime} \check{\succsim}_{c} S^{\prime} \backslash S^{\prime \prime} \cup\{s\}$. However, this contradicts assumption $S^{\prime} \backslash S^{\prime \prime} \cup\{s\} \tilde{\succ}_{c} S^{\prime}$.

Lemma 5. Assume $Y$ is the obtained matching of $S D A$, where for student $s$, where $s \succ_{c} s_{\emptyset}$, contract $(s, c, w)$ is rejected. Let $Z=\left\{\left(s^{\prime}, c, w^{\prime}\right) \in Y_{c} \mid w^{\prime} \geq w\right\}$. Then $b_{c}-\sum_{\left(s^{\prime}, c, w^{\prime}\right) \in Z} w^{\prime}<$ $w$ holds.

In other words, if contract $(s, c, w)$ is rejected although college $c$ prefers to have student $s$, then $c$ does not have enough money in its budget to accept the contract even when $c$ rejects all of the contracts whose weights are less than $w$ (note that $b_{c}-\sum_{\left(s^{\prime}, c, w^{\prime}\right) \in Z} w^{\prime}$ is the remaining money when only contracts whose weights are more than or equal to $w$ are accepted, i.e., the rest of contracts are rejected).

Proof of Lemma 5. Each student $s^{\prime}$, whose type is $\theta$, proposes $\left(s^{\prime}, c, w^{\prime}\right)$ only after she has proposed $\left(s^{\prime}, c, w^{\prime \prime}\right)$ (and it is rejected) for all $w^{\prime \prime} \in W_{c, \theta}$ such that $w^{\prime \prime}>w^{\prime}$ holds. Thus the fact that $(s, c, w)$ is rejected implies that there exists contract $\left(s^{\prime}, c, w\right)$ where $s^{\prime} \succ_{c} s_{\emptyset}$ that was rejected while no contract whose weight is less than $w$ has been proposed yet by a student whom college $c$ prefers to $s_{\emptyset}$ (here, $s^{\prime}$ can be $s$, i.e., $(s, c, w)$ is the first contract rejected with weight $w$, or $s^{\prime}$ can be different from $s$, i.e., $\left(s^{\prime}, c, w\right)$ is rejected before $\left.(s, c, w)\right)$.

Thus all of the contracts accepted so far have weights larger than or equal to $w$. Then $b_{c}-\sum_{\left(s^{\prime}, c, w^{\prime}\right) \in Z} w^{\prime}<w$ must hold.

Proof of Theorem \%. Assume by way of contradiction that blocking pair $(s, c)$ exists for obtained matching $Y$. More precisely, we assume $R \subseteq Y_{c}$ and $w \in W_{c, \tau(s)}$ exist such that (i) $(c, w) \succ_{s} Y(s)$, (ii) $(Y(c) \backslash R(c)) \cup\{s\} \tilde{\succ}_{c} Y(c)$, and (iii) $\sum_{x \in Y_{c} \backslash R} x_{W}+w \leq b_{c}$ hold. From (i), $s$ must have proposed $(s, c, w)$, which was rejected. Then by Lemma 5 , we have $b_{c}-\sum_{\left(s^{\prime}, c, w^{\prime}\right) \in Z} w^{\prime}<w$, where $Z=\left\{\left(s^{\prime}, c, w^{\prime}\right) \in Y_{c} \mid w^{\prime} \geq w\right\}$ and we obtain:

$$
b_{c}<\sum_{\left(s^{\prime}, c, w^{\prime}\right) \in Z} w^{\prime}+w
$$


From (ii) and Lemma 4, we have $\forall s^{\prime} \in R(c), s \succ_{c} s^{\prime}$. Then for all $s^{\prime} \in R(c)$, where $\left(s^{\prime}, c, w^{\prime}\right) \in Y_{c}, w^{\prime}<w$ holds (otherwise, $(s, c, w)$ must be accepted instead of $\left.\left(s^{\prime}, c, w^{\prime}\right)\right)$. Thus $\sum_{x \in Y_{c} \backslash R} x_{W} \geq \sum_{\left(s^{\prime}, c, w^{\prime}\right) \in Z} w^{\prime}$ holds, since $Y_{c} \backslash R \supseteq Z$. Combined with (iii), we obtain

$$
\begin{aligned}
b_{c} & \geq \sum_{x \in Y_{c} \backslash R} x_{W}+w \\
& \geq \sum_{\left(s^{\prime}, c, w^{\prime}\right) \in Z} w^{\prime}+w
\end{aligned}
$$

which contradicts (1).

\subsection{Strategy-Proofness of SDA}

Theorem 8. SDA is strategy-proof for students.

Proof. Assume student $s$ is a type $\theta_{i}$ student, i.e., she is assigned in Round $i$. Student $s$ clearly has no influence on the outcomes of Round $j$, where $j<i$. Also, the outcome of the later rounds is irrelevant to $i$. Thus, to show the strategy-proofness of SDA, it is sufficient to show the strategy-proofness of DA used for each round. To show this fact, we introduce an alternative market in which each (sub-)college has its maximum quota/capacity limit (but no budget constraints). In this market, DA is guaranteed to be strategy-proof. We show the equivalence of the outcomes in these markets.

The alternative market is defined as follows. Assume $W_{c, \theta_{i}}$, i.e., the possible set of $c$ 's weights for type $\theta_{i}$ students, is given as $\left\{w_{c}^{1}, w_{c}^{2}, \ldots, w_{c}^{\ell_{c}}\right\}$, where $w_{c}^{1}>\ldots>w_{c}^{\ell_{c}}$ for all $c \in C$. We divide college $c$ into $\ell_{c}$ sub-colleges, i.e., $c^{1}, c^{2}, \ldots, c^{\ell_{c}}$. Maximum quota $q_{c^{i}}$ for each sub-college $c^{i}$ is recursively defined as follows, where $r_{1}=b_{c}$ (more precisely, $b_{c}$ is the budget amount obtained in each round of SDA):

$$
q_{c^{i}}=\left\lfloor r_{i} / w_{c}^{i}\right\rfloor, r_{i+1}=r_{i}-q_{c^{i}} \times w_{c}^{i} .
$$

Contract $\left(s, c, w_{c}^{i}\right)$ in the original market is translated into contract $\left(s, c^{i}\right)$ in the alternative market. The preference of each student in the alternative market is identical to the original market based on the above translation. The preference of each sub-college $c^{i}$ is defined based on $\succsim_{c}$; i.e., $c^{i}$ will accept students according to $\succsim_{c}$ until satisfying its maximum quota $q_{c^{i}}$, using the same tie-breaking method as $C h_{c}$.

In the original market, $c$ can accept at most $q_{c^{1}}$ contracts with weight $w_{c}^{1}$ due to its budget constraints. Also, each student $s$ proposes contract $\left(s, c, w_{c}^{2}\right)$ only after $\left(s, c, w_{c}^{1}\right)$ is rejected. This implies that $c$ already accepts $q_{c^{1}}$ contracts with weight $w_{c}^{1}$. Then $c$ can accept at most $q_{c^{2}}$ contracts with weight $w_{c}^{2}$ due to its budget constraints. Also, each student $s$ proposes contract $\left(s, c, w_{c}^{3}\right)$ only after $\left(s, c, w_{c}^{2}\right)$ is rejected. This implies that $c$ accepts $q_{c^{2}}$ contracts with weight $w_{c}^{2}$, and so on. From these facts, the outcomes in both the alternative and original markets must be identical. Then from the fact that the standard DA in the alternative market is strategy-proof, the DA (Definition 15) in the original market must be strategy-proof.

Let us show an example of the alternative market using the original market illustrated in Fig. 1. In Round 1, we create two sub-colleges for $c_{1}$, i.e., $c_{1}^{1}$ and $c_{1}^{2}$, whose maximum 
quotas are 1 . Only one sub-college exists for $c_{2}$, which we denote as $c_{2}^{1}$, whose maximum quota is also 1. Then $s_{1}$ is accepted for $c_{1}^{1}, s_{2}$ is accepted for $c_{1}^{2}$, and $s_{3}$ is accepted for $c_{2}^{1}$. In Round 2, since the sub-college for $c_{1}$ has no capacity, its maximum quota is 0 . There exists one sub-college for $c_{2}$, which we denote as $c_{2}^{1}$, whose maximum quota is 2 . Then, $s_{4}$ and $s_{5}$ are accepted for $c_{2}^{1}$.

From Theorem 7, we immediately obtain the following.

Theorem 9. In a typed weighted market, a pairwise stable matching is guaranteed to exist and can be calculated in time linear in $|X|$, assuming $C h_{C}$ and $C h_{\hat{S}}$ can be calculated in constant time.

Proof. We can always find a pairwise stable matching using SDA. Also, during the iteration of DA in Definition 15, at least one contract must be rejected; otherwise, the procedure is terminated. Thus, assuming $C h_{C}$ and $C h_{\hat{S}}$ can be calculated in a constant time, the run-time of SDA is linear in $|X|$.

A standard way to prove the fact that a DA-based mechanism is strategy-proofness is to utilize the result obtained by (Hatfield \& Milgrom, 2005). More specifically, Hatfield \& Milgrom (2005) show that when the choice functions of all colleges satisfy the following three properties, DA is guaranteed to be strategy-proof for students. Informally, the irrelevance of rejected contracts means if contract $x$ is rejected when it is added to $X^{\prime}$, it does not affect the outcomes of the other contracts in $X^{\prime}$. Also, substitutability means if some contract $x$ is rejected when $x \in X^{\prime}$, it is also rejected when another contract is added to $X^{\prime}$. Furthermore, the law of aggregate demand means if the set of contracts expands, the number of accepted contracts weakly increases.

Note that we cannot apply this result to SDA. Although our choice functions satisfy the irrelevance of rejected contracts, they fail to satisfy the remainder. For example, assume four students, s.t. $s_{1} \succ_{c} s_{2} \succ_{c} s_{3} \succ_{c} s_{4}$, and $b_{c}=5$. From $\left\{\left(s_{2}, c, 2\right),\left(s_{3}, c, 3\right),\left(s_{4}, c, 1\right)\right\}$, contract $\left(s_{4}, c, 1\right)$ is rejected. However, from $\left\{\left(s_{1}, c, 2\right),\left(s_{2}, c, 2\right),\left(s_{3}, c, 3\right),\left(s_{4}, c, 1\right)\right\},\left(s_{4}, c, 1\right)$ is accepted. Thus, substitutability is violated. Also, from contracts $\left\{\left(s_{2}, c, 2\right),\left(s_{3}, c, 2\right),\left(s_{4}, c, 1\right)\right\}$, all three contracts are accepted. However, from $\left\{\left(s_{1}, c, 3\right),\left(s_{2}, c, 2\right),\left(s_{3}, c, 2\right),\left(s_{4}, c, 1\right)\right\}$, only the first two contracts are accepted. Thus, the law of aggregated demand is violated.

In (Fleiner \& Jankó, 2014), it is shown that even if the choice function does not satisfy the irrelevance of rejected contracts (which they call path-independence), a pairwise stable matching is guaranteed to exist in some cases. The cases discussed in (Fleiner \& Jankó, 2014) include the weighted scoring choice function, which might look similar to our choice function, but they are completely different. The weighted scoring choice function satisfies substitutability, while it violates the irrelevance of rejected contracts. Our choice function satisfies the irrelevance of rejected contracts, while it violates the substitutability. This difference comes from the fact that in the weighted scoring choice function, a college cannot skip some contracts and accept a lower ranked contract with a lower wage. For example, from $\left\{\left(s_{1}, c, 2\right),\left(s_{2}, c, 2\right),\left(s_{3}, c, 3\right),\left(s_{4}, c, 1\right)\right\}$, the weighted scoring choice function can accept $\left(s_{1}, c, 2\right)$ and $\left(s_{2}, c, 2\right)$, while it cannot accept $\left(s_{3}, c, 3\right)$ due to the budget constraint. Then, it is not allowed to accept $\left(s_{4}, c, 1\right)$, even though the college can afford to accept it. Thus, our results are logically independent from the results obtained in (Fleiner \& Jankó, 2014). 


\subsection{Constrained Pareto Efficiency}

In this subsection, we consider the class of typed weighted markets where the preference of each college over the students is strict, that is, $\hat{\succsim}_{c}$ is based on a strict preference $\succ_{c}$ over the individual students and $s_{\emptyset}$ for each $c$. In this class of markets, we show that SDA is constrained Pareto efficient. That is, its outcome is Pareto efficient among the pairwise stable matchings.

First, let us introduce several notations. For $1 \leq \ell<k$, let $\Theta^{-\ell}$ denote $\left\{\theta_{i} \mid \theta_{i} \in \Theta, \ell<\right.$ $i \leq k\}, S^{-\ell}$ denote $\left\{s \mid s \in S, \tau(s) \in \Theta^{-\ell}\right\}$, and $X_{S^{-\ell}}$ denote $\left\{\underset{\sim}{x} \mid x \in X, x_{S} \in S^{-\ell}\right\}$. For typed weighted market $\pi=\left(S, C, \Theta, \tau,\left(W_{c, \theta}\right)_{c \in C, \theta \in \Theta}, X, b_{C}, \succ_{S}, \tilde{\succsim}_{C}\right)$ and matching $Y$, let

$$
\pi^{-\ell, Y}=\left(S^{-\ell}, C, \Theta^{-\ell}, \tau, \quad\left(W_{c, \theta}\right)_{c \in C, \theta \in \Theta^{-\ell}}, X_{S^{-\ell}}, \quad\left(b_{c}^{-\ell, Y}\right)_{c \in C}, \quad\left(\succ_{s}\right)_{s \in S^{-\ell}}, \tilde{\succsim}_{C}\right)
$$

denote the reduced market of $\pi$ by $Y$ until $\ell$, where

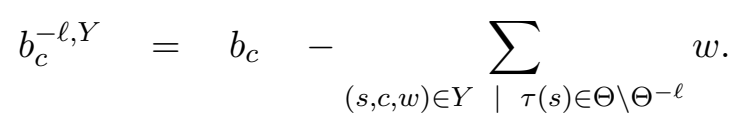

In other words, the reduced market of $\pi$ by $Y$ until $\ell$ is a market where all the students higher than or equal to type $\theta_{\ell}$ are removed and the budget of each college is reduced by the wages of the removed students in $Y$. Finally, for a given set of contracts $Y$ and $1 \leq i \leq k$, let $Y^{i}=\left\{x \in Y \mid \tau\left(x_{S}\right)=\theta_{i}\right\}$, i.e., the set of contracts of students with type $\theta_{i}$ in $Y$.

First, we show that we can safely combine a subset of the matching obtained by SDA and the matching for the reduced market.

Lemma 6. Let $Y=\bigcup_{1 \leq i \leq k} Y^{i}$ be the matching obtained by SDA for a typed weighted market $\pi$, and for some $1 \leq \ell<k$, assume $Z^{-\ell}$ is a pairwise stable matching for reduced market $\pi^{-\ell, Y}$. Then $\bigcup_{1 \leq i \leq \ell} Y^{i} \cup Z^{-\ell}$ is feasible and pairwise stable for $\pi$.

Proof. Set of contracts $\bigcup_{1 \leq i \leq \ell} Y^{i} \cup Z^{-\ell}$ is feasible for $\pi$ because (i) clearly every student is matched with at most one contract and (ii) the budget of each college $c$ is satisfied due to the fact that $\sum_{1 \leq i \leq \ell} \sum_{(s, c, w) \in Y_{c}^{i}} w=b_{c}-b_{c}^{-\ell, Y}$ and $\sum_{(s, c, w) \in Z_{c}^{-\ell}} w \leq b_{c}^{-\ell, Y}$. Regarding pairwise stability, suppose there is a blocking pair $(s, c)$ with $\tau(s)=\theta_{i}$. First, assume $\ell<i$. From the assumption of the typed weighted market, a college will never form a blocking pair with a lower-typed student to reject a higher-typed student. Thus $c$ must prefer to reject some contracts in $Z_{c}^{-\ell}$ in order to accept $s$, which contradicts that $Z^{-\ell}$ is pairwise stable for $\pi^{-\ell, Y}$. Next, assume $\ell \geq i$. From Lemma 5,c cannot save enough money to accept $s$ even if $c$ rejects all students in $Z_{c}^{-\ell}$. Thus $c$ must prefer to reject some contracts in $\bigcup_{1 \leq i \leq \ell} Y_{c}^{i}$ in order to accept $s$, which contradicts that $Y$ is pairwise stable for $\pi$.

We use the following lemmas to prove that SDA is constrained Pareto efficient. We proceed by induction on $\ell$ : Lemma 7 is the induction base, Lemma 8 is the induction step.

Lemma 7. Let $\pi$ be a typed weighted market where the preference of each college over the individual students is strict. Let $Y=\bigcup_{1 \leq i \leq k} Y^{i}$ be the matching obtained by SDA for $\pi$, and let $Z^{-1}$ be any constrained Pareto efficient matching for reduced market $\pi^{-1, Y}$. Then matching $Y^{1} \cup Z^{-1}$ is constrained Pareto efficient for $\pi$. 
Proof. From Lemma 6, it follows that $Y^{1} \cup Z^{-1}$ is pairwise stable. By way of contradiction, assume that another pairwise stable matching $\widehat{Z}=\bigcup_{1 \leq i \leq k} \widehat{Z}^{i}$ Pareto dominates $Y^{1} \cup Z^{-1}$. Let $S^{\prime}$ denote the set of students who strictly prefer $\widehat{Z}$ over $Y^{1} \cup Z^{-1}$. Assume $\bigcup_{c \in C} Y^{1}(c)$ intersects with $S^{\prime}$. This implies that $\widehat{Z}^{1}$ Pareto dominates $Y^{1}$ and there is no blocking pair $(s, c)$ with $\tau(s)=\theta_{1}$ for $\widehat{Z}^{1}$. However, since $Y^{1}$ is identical to the outcome of the standard DA for type $\theta_{1}$ students and preferences of both students and colleges are strict, this contradicts the student-optimality of the standard DA (Gale \& Shapley, 1962). Thus $S^{\prime} \subseteq S^{-1}$ holds. Then $Y^{1}$ and $\widehat{Z}^{1}$ must be identical. Thus $\widehat{Z}^{-1}$ Pareto dominates $Z^{-1}$. However, this is a contradiction to the assumption that $Z^{-1}$ is constrained Pareto efficient.

Lemma 8. Let $\pi$ be a typed weighted market where the preference of each college over the individual students is strict. Let $Y=\bigcup_{1 \leq i \leq k} Y^{i}$ be the matching obtained by SDA for $\pi$. For any $\ell$ s.t. $1 \leq \ell<k$, assume for any $Z^{-\ell}$, which is a constrained Pareto efficient matching for reduced market $\pi^{-\ell, Y}$, that $\bigcup_{1 \leq i \leq \ell} Y^{i} \cup Z^{-\ell}$ is constrained Pareto efficient for market $\pi$. Then for any $Z^{-(\ell+1)}$, which is a constrained Pareto efficient matching for reduced market $\pi^{-(\ell+1), Y}, \bigcup_{1 \leq i \leq \ell+1} Y^{i} \cup Z^{-(\ell+1)}$ is also constrained Pareto efficient.

Proof. Assume the assumption is true, i.e., for any $Z^{-\ell}$, which is a constrained Pareto efficient matching for reduced market $\pi^{-\ell, Y}, \bigcup_{1 \leq i \leq \ell} Y^{i} \cup Z^{-\ell}$ is constrained Pareto efficient. Then as for Lemma 7 , for any $Z^{-(\ell+1)}$, which is a constrained Pareto efficient matching for reduced market $\pi^{-(\ell+1), Y}$, we can show that $Y^{\ell+1} \cup Z^{-(\ell+1)}$ is a constrained Pareto efficient matching for reduced market $\pi^{-\ell, Y}$. Thus, from the assumption, $\bigcup_{1 \leq i \leq \ell} Y^{i} \cup Y^{\ell+1} \cup Z^{-(\ell+1)}$, which equals $\bigcup_{1 \leq i \leq \ell+1} Y^{i} \cup Z^{-(\ell+1)}$, is constrained Pareto efficient.

Theorem 10. Let $\pi$ be a typed weighted market where the preference of each college over the individual students is strict. SDA obtains a constrained Pareto efficient matching for $\pi$.

Proof. Let $Y=\bigcup_{1 \leq i \leq k} Y^{i}$ be the matching obtained by SDA. From Lemma 7, it holds that $Y^{1} \cup Z^{-1}$ is constrained Pareto efficient for any $Z^{-1}$, which is a constrained Pareto efficient matching for $\pi^{-1, Y}$. Then by repeatedly applying Lemma 8, we obtain $Y^{1} \cup Y^{2} \cup \ldots \cup Y^{k}=Y$ is constrained Pareto efficient.

If the preference of each college over the individual students is not strict, SDA uses some tie-breaking method to determine the order among indifferent students as described in Definition 14. According to this tie-breaking method, there is a chance that another pairwise stable matching Pareto dominates the outcome of SDA. A similar situation also occurs in a standard two-sided matching problem when the preference of a college is not strict (Abdulkadiroğlu et al., 2009).

\section{Conclusion}

This paper examined two-sided matchings with budget constraints and showed several computational hardness results for problems related to coalitional stability. Then, we designed a strategy-proof mechanism, which is pairwise stable and Pareto efficient among the pairwise stable outcomes. 
Our future work include examining the complexity when the number of types is limited (we suspect problem CSWM to be easier for a constant number of types), examining a broader market than a typed market, in which we can guarantee the existence of some stability requirement and obtain a strategy-proof mechanism. It would also be interesting to study the case where a wage can be determined via negotiation between students and colleges.

\section{Acknowledgment}

This work was partially supported by JSPS KAKENHI Grant Numbers 24220003 and 17H00761. This paper is based on our conference publication (Hamada, Ismaili, Suzuki, \& Yokoo, 2017). The main differences are found in Sections 3 and 4 . We provide extended proofs and generalize our complexity results to strict preferences for colleges and to coalitions with a bounded size. Section 4 is extended and includes a proof of constrained Pareto efficiency.

\section{References}

Abdulkadiroğlu, A. (2005). College admissions with affirmative action. International Journal of Game Theory, 33(4), 535-549.

Abdulkadiroğlu, A., Pathak, P. A., \& Roth, A. E. (2009). Strategy-proofness versus efficiency in matching with indifferences: Redesigning the NYC high school match. American Economic Review, 99(5), 1954-1978.

Abdulkadiroğlu, A., Pathak, P. A., Roth, A. E., \& Sönmez, T. (2005). The Boston public school match. American Economic Review P\&SP, 95, 368-371.

Abdulkadiroğlu, A., \& Sönmez, T. (2003). School choice: A mechanism design approach. American Economic Review, 93(3), 729-747.

Abizada, A. (2016). Stability and incentives for college admissions with budget constraints. Theoretical Economics, 11, 735-756.

Aziz, H., Brandt, F., \& Seedig, H. G. (2013). Computing desirable partitions in additively separable hedonic games. Artificial Intelligence, 195, 316-334.

Biró, P., Fleiner, T., Irving, R. W., \& Manlove, D. F. (2010). The college admission problem with lower and common quotas. Theoretical Computer Science, 411, 3136 - 3153.

Bouveret, S., \& Lang, J. (2008). Efficiency and envy-freeness in fair division of indivisible goods: Logical representation and complexity. Journal of Artificial Intelligence Research, 32, 525-564.

Echenique, F. (2012). Contracts vs. salaries in matching. American Economic Review, 102(1), 594-601.

Ehlers, L., Hafalir, I. E., Yenmez, M. B., \& Yildirim, M. A. (2014). School choice with controlled choice constraints: Hard bounds versus soft bounds. Journal of Economic Theory, 153, 648-683.

Fleiner, T., \& Jankó, Z. (2014). Choice function-based two-sided markets: Stability, lattice property, path independence and algorithms. Algorithms, 7(1), 32-59. 
Fragiadakis, D., Iwasaki, A., Troyan, P., Ueda, S., \& Yokoo, M. (2015). Strategyproof matching with minimum quotas. ACM Transactions on Economics and Computation, $4(1), 6: 1-6: 40$.

Fragiadakis, D., \& Troyan, P. (2017). Improving matching under hard distributional constraints. Theoretical Economics., 12(2), 863-908.

Gale, D., \& Shapley, L. S. (1962). College admissions and the stability of marriage. The American Mathematical Monthly, 69(1), 9-15.

Goto, M., Iwasaki, A., Kawasaki, Y., Kurata, R., Yasuda, Y., \& Yokoo, M. (2016). Strategyproof matching with regional minimum and maximum quotas. Artificial Intelligence, 235, 40-73.

Hamada, N., Ismaili, A., Suzuki, T., \& Yokoo, M. (2017). Weighted matching markets with budget constraints. In Proceedings of the 16th International Conference on Autonomous Agents and MultiAgent Systems (AAMAS-2017), pp. 317-325.

Hatfield, J. W., \& Kojima, F. (2008). Matching with contracts: Comment. American Economic Review, 98, 1189-1194.

Hatfield, J. W., \& Kojima, F. (2010). Substitutes and stability for matching with contracts. Journal of Economic Theory, 145, 1704-1723.

Hatfield, J. W., \& Kominers, S. D. (2009). Contract design and stability in matching markets. mimeo.

Hatfield, J. W., \& Kominers, S. D. (2012). Matching in networks with bilateral contracts. American Economic Journal: Microeconomics, 176-208.

Hatfield, J. W., Kominers, S. D., Nichifor, A., Ostrovsky, M., \& Westkamp, A. (2013). Stability and competitive equilibrium in trading networks. Journal of Political Economy, $121(5), 966-1005$.

Hatfield, J. W., \& Milgrom, P. R. (2005). Matching with contracts. American Economic Review, 95(4), 913-935.

Huang, C.-C. (2010). Classified stable matching. In Proceedings of the 21st ACM-SIAM Symposium on Discrete Algorithms (SODA-2010), pp. 1235-1253.

Kamada, Y., \& Kojima, F. (2015). Efficient matching under distributional constraints: Theory and applications. American Economic Review, 105(1), 67-99.

Karp, R. M. (1972). Reducibility among combinatorial problems. In Complexity of computer computations, pp. 85-103. Springer.

Kawase, Y., \& Iwasaki, A. (2017). Near-feasible stable matchings with budget constraints. In Proceedings of the 26th International Joint Conference on Artificial Intelligence (IJCAI-2017), pp. 242-248.

Kawase, Y., \& Iwasaki, A. (2018). Approximately stable matchings with budget constraints. In Proceedings of the 32nd AAAI Conference on Artificial Intelligence (AAAI-2018), pp. 1113-1120.

Kojima, F. (2012). School choice: Impossibilities for affirmative action. Games and Economic Behavior, 75(2), 685-693. 
Kojima, F., Tamura, A., \& Yokoo, M. (2018). Designing matching mechanisms under constraints: An approach from discrete convex analysis. Journal of Economic Theory, $176,803-833$.

Kominers, S. D., \& Sönmez, T. (2016). Matching with slot-specific priorities: Theory. Theoretical Economics, 11(2), 683-710.

Kurata, R., Hamada, N., Iwasaki, A., \& Yokoo, M. (2017). Controlled school choice with soft bounds and overlapping types. Journal of Artificial Intelligence Research, 58, $153-184$.

Meyer, A. R., \& Stockmeyer, L. J. (1972). The equivalence problem for regular expressions with squaring requires exponential space. In Proceedings of the 13th Annual Symposium on Switching and Automata Theory (SWAT-1972), pp. 125-129.

Mongell, S. J., \& Roth, A. E. (1986). A note on job matching with budget constraints. Economics Letters, $21(2), 135$ - 138.

Perrault, A., Drummond, J., \& Bacchus, F. (2016). Strategy-proofness in the stable matching problem with couples. In Proceedings of the 15th International Conference on Autonomous Agents and Multiagent Systems (AAMAS-2016), pp. 132-140.

Ronn, E. (1990). NP-complete stable matching problems. Journal of Algorithms, 11(2), $285-304$.

Roth, A. E. (1984). The evolution of the labor market for medical interns and residents: A case study in game theory. Journal of Political Economy, 92(6), 991-1016.

Roth, A. E., \& Peranson, E. (1999). The redesign of the matching market for american physicians: Some engineering aspects of economic design. American Economic Review, 89(4), 748-780.

Roth, A. E., \& Sotomayor, M. A. O. (1990). Two-sided Matching: a Study in Game-theoretic Modeling and Analysis. Econometric Society Monographs, Cambridge.

Schaefer, M., \& Umans, C. (2002). Completeness in the polynomial-time hierarchy: A compendium. SIGACT news, 33(3), 32-49.

Sönmez, T. (2013). Bidding for army career specialties: Improving the ROTC branching mechanism. Journal of Political Economy, 121(1), 186-219.

Sönmez, T., \& Switzer, T. B. (2013). Matching with (branch-of-choice) contracts at the united states military academy. Econometrica, 81(2), 451-488.

Sung, S. C., \& Dimitrov, D. (2007). On core membership testing for hedonic coalition formation games. Operations Research Letters, 35(2), 155-158.

Woeginger, G. J. (2013). A hardness result for core stability in additive hedonic games. Mathematical Social Sciences, 65(2), 101-104.

Yenmez, M. B., Yildirim, M. A., \& Hafalir, I. E. (2013). Effective affirmative action in school choice. Theoretical Economics, 8, 325-363. 